\title{
The Exponential Transform: A Renormalized Riesz Potential at Critical Exponent
}

\author{
BJÖRn Gustafsson \& Mihai Putinar
}

\begin{abstract}
For an arbitrary domain in $\mathbb{R}^{n}$ we consider the exponential of a suitably normalized Riesz potential of first nonintegrable index. This gives a positive function, with certain monotonicity properties, defined in the complement of the domain, and vanishing on its boundary. In two dimensions this function, polarized into two complex variables, has previously been studied and proved to be useful within operator theory, moment problems and other problems of domain identification, and for proving regularity of free boundaries.

Even in the absence of a natural polarization there are noteworthy properties of the higher dimensional exponential transform. For instance we show that this function is superharmonic and that it tends to zero at smooth points of the boundary, with slope bounded from above and below by constants depending solely on the local curvature. The analytic continuation configuration of the exponential transform of a convex polyhedron shows that it is indeed a natural defining function of its boundary. In addition, computations of this transform are carried out in full detail for some domains bounded by lower degree algebraic surfaces.

There is also an inner exponential transform, defined inside the domain. If the domain is convex then the ordinary Newtonian potential of it can, for points inside the domain, be interpreted is an arithmetic meanvalue with respect to the solid angle of the squared distance to the boundary. The interior exponential transform is then the corresponding geometric meanvalue.
\end{abstract}




\section{INTRODUCTION}

The exponential transform (in the terminology adopted throughout this paper) or the related integral transform of a domain in the complex plane has proved to be a remarkable object within branches of mathematics such as function theory [4], [13], operator theory [18], [5], see also [17], moment problems [19], [20], inverse recovery problems [12], analytic continuation [11], free boundary problems [10] and so forth. A corresponding exponential transform on the real axis was already known and used by A.A. Markov (in the 19th century) and later by N.I. Akhiezer and M.G. Krein in their studies of one-dimensional moment problems [3], [15].

In the present paper we take some first steps towards generalizing the exponential transform to higher real dimensions. Our aim is mainly to set the stage, i.e., to introduce appropriate concepts, find out basic properties of these and elaborate a number of examples. We are all the time guided by the highly successful two-dimensional case.

The exponential transform can be viewed as a potential depending on a domain in $\mathbb{R}^{n}$, or more generally on a measure having a density function $\rho$ in the range $0 \leq \rho \leq 1$. The two-dimensional version of it is, in case $\chi_{\Omega}, \Omega \subset \mathbb{C}=\mathbb{R}^{2}$,

$$
E_{\Omega}(z, w)=\exp \left[-\frac{1}{\pi} \int_{\Omega} \frac{d A(\zeta)}{(\zeta-z)(\bar{\zeta}-\bar{w})}\right] .
$$

It is defined for all $z, w \in \mathbb{C}$ but have different behaviour depending on whether $z$ and $w$ are inside or outside $\Omega$. Inside $\Omega$ the function

$$
H_{\Omega}(z, w)=-\frac{\partial^{2} E_{\Omega}(z, w)}{\partial \bar{z} \partial w}
$$

is perhaps more interesting than $E_{\Omega}$ itself. It is analytic in $z$, antianalytic in $w$. The same is true for $E_{\Omega}(z, w)$ when the variables are outside $\Omega$.

What we generalize in this paper is the diagonal version of (1.1), namely $E_{\Omega}(z, z)$. This gives us

$$
E_{\Omega}(x)=\exp \left[-\frac{2}{\left|S^{n-1}\right|} \int_{\Omega} \frac{d y}{|x-y|^{n}}\right],
$$

the (exterior) exponential transform of $\Omega \subset \mathbb{R}^{n}$. It is of interest only for $x \in \mathbb{R}^{n} \backslash$ $\Omega$. We have not found any natural polarization of it into two (or more) variables, corresponding to $E_{\Omega}(z, w)$, but we still have a version of it defined inside the domain, the interior exponential transform $H_{\Omega}(x)$ generalizing $H_{\Omega}(z, z)$. It can for example be defined as one over a rescaled exterior exponential transform of the complementary domain:

$$
H_{\Omega}(x)=\lim _{R \rightarrow \infty} \frac{1}{R^{2} E_{B_{R} \backslash \Omega}(x)} \quad(x \in \Omega),
$$


$B_{R}$ denoting the ball of radius $R$.

One may notice that the exponential transform (1.2) is the exponential of a Riesz potential at critical index, "critical" referring to that the power $n$ occurring in the kernel is the first one making it not locally integrable. Traditionally one considers Riesz kernels $1 /|x|^{n-\alpha}$ mainly for $\alpha>0$. The leading term after integration of the critical Riesz kernel is a logarithmic one, and therefore the right way to tame the singularity (or "renormalize" it) is to take the exponential, with a carefully selected constant, namely $-2 /\left|S^{n-1}\right|$. Thus we arrive at (1.2).

So we may conceive the higher dimensional exponential transform as a border case of classical Riesz potentials. The original philosophy of M.Riesz [21], [22] for introducing his potentials was to solve by means of explicit formulas certain partial differential equations and to generalize the classical potential theory. More recent applications concern e.g. inversion of Radon type transforms (geometric tomography) [14], [8]. This connects with directions in which the exponential transform seems to be powerful: domain identification from gravimetric data, moment data, tomographic data and similar. Also certain regularity questions for free boundaries, at least in two dimensions, can be effectively handled by the exponential transform [10].

The reason that the exponential transform is linked to domain identification is that it (like most of the Riesz potentials) is uniquely determined by its germ at infinity, hence by its moments, and that it tends to zero at $\partial \Omega$ with nonzero slope (generically). Therefore, in case it has a (say) real analytic extension across $\partial \Omega$ this extended function will be a good defining function for $\partial \Omega$. Hence it makes sense in principle to try to identify $\partial \Omega$ from e.g. moment data by using the exponential transform.

This theoretical programme really leads to effective algorithms, as has been demonstrated in one (classical moment theory [3], [15]) and two (more recent [19], [20], [12]) dimensions. The idea in these lower dimensional cases is that there is a dense set of data ("degenerate moment sequences" in case of moment data) for which $E_{\Omega}$ is a rational function of some special form, that it is possible already from a finite initial segment of these data to recognize that you are in such a case (and if not you can by approximation or otherwise arrange to be), and from that on you can effectively reconstruct everything (the rational function, $\partial \Omega$ etc.).

In dimension $n \geq 3$ programmes of this type remain to be elaborated and more theoretical studies may be necessary. Thus the present paper just provides a first step in a hopefully useful direction, and several important questions remain open.

The contents of the paper is as follows. In Section 2 we introduce the exponential transform in a rather general setting by starting from the Riesz kernel $1 /|x|^{n-\alpha}, \alpha>0$, and letting $\alpha \rightarrow 0$ in combination with the appropriate renormalizations. This gives a somewhat lengthy tour leading to the after all rather simple definitions as stated above (and in more complete form in Definition 2.1). This long tour has the advantage that the two versions of the exponential transform, the exterior and the interior one, arise simultaneously as one single object. In (2.7), for example, they are written in one formula. 
In Section 3 we use Stokes' formula to write the volume integrals appearing in the exponential transforms as boundary integrals. From this it becomes even more transparent that the exterior and interior transforms are really the same thing: they are both given by

$$
\exp \left[-\frac{2}{\left|S^{n-1}\right|} \int_{\partial \Omega} \log |x-y| d \theta(y-x)\right],
$$

where $d \theta$ denotes the solid angle $(n-1)$-form. The difference between the exterior and interior transforms here comes from the difference in sign appearing when $\partial \Omega$ is viewed from outside and inside.

One interesting thing with the above expression is that it can be interpreted as a geometric mean of distances $|x-y|$ (to some power) with respect to $d \theta(y-x)$ in cases in which the latter can be viewed as a positive measure (e.g., when $\Omega$ is convex and $x \in \Omega$ ). The ordinary Newtonian potential of $\Omega$ turns out to be a corresponding arithmetic mean value, so if one considers ordinary potential theory as a linear or additive theory, the theory for the exponential transform will be a corresponding multiplicative theory. Notice however that we only deal with a rather restricted potential theory, namely for bodies of constant density.

In Section 4 we compute the exponential transform in some simple cases, mainly for domains bounded by quadratic or lower degree surfaces. Perhaps the most interesting case here is that the interior transform for an ellipsoid or paraboloid equals a constant over the defining polynomial (Theorem 4.4). For example,

$$
H(x)=\frac{1}{1-|x|^{2}}
$$

for the unit ball in any number of dimensions.

Section 5 concerns boundary behaviour. It is shown that under suitable inner and outer ball conditions $E_{\Omega}$ is Lipschitz continuous and tends to zero at $\partial \Omega$ as the distance function. The interior transform $H_{\Omega}$, on the other hand, tends to plus infinity as one over the distance to the boundary.

We have not investigated in general higher order regularity $\left(C^{1}, C^{\infty}\right.$ etc. $)$ of $E_{\Omega}$ up to $\partial \Omega$ in case $\partial \Omega$ is correspondingly smooth. An issue of special importance and interest, however, is smoothness in the real analytic category up to the boundary. In two dimensions it is known [20] that if $\partial \Omega$ is smooth real analytic, then $E_{\Omega}$ has a real analytic extension across $\partial \Omega$. The most striking result in this direction states that the polarized transform (1.1) has an extension, analytic in $z$, antianalytic in $w$, across $\partial \Omega$ if and only if the exterior Cauchy transform $\hat{\chi}_{\Omega}$ of $\Omega$ has an analytic extension across $\partial \Omega$. Moreover, the analytic extensions are related via explicit formulas and propagate down to the same singularity set [10], [11]. These matters are reviewed and put in new perspectives in Section 6, with an eye to possible generalizations to higher dimensions.

In [10] the analytic extension of $E_{\Omega}$ was used to prove apriori regularity of some free boundaries (those which admit analytic continuation of $\hat{\chi}_{\Omega}$ ) and one 
of our original hopes with the higher dimensional exponential transform was that something similar could be done in higher dimension. However, whether this can be done remains open, and we have so far only limited results concerning analytic continuation in higher dimensions, most of them not included in this paper. One particular case in which analytic continuation is easily established is when the boundary is perfectly flat.

In Section 7 we use this to obtain explicit analytic continuation formulas in the case of convex polyhedra. The rather complete picture we offer for this basic class of domains invites to use the exponential transform in reconstruction algorithms following a multivariate Padé type approximation scheme. We will resume this subject in a separate paper.

In Section 8, finally, we prove that $E_{\Omega}$ and $1 / H_{\Omega}$ are superharmonic functions. In two dimensions it is also known that $\log \left(1-E_{\Omega}\right)$ is subharmonic, which is a stronger statement. That result fails in higher dimensions, but we still suspect, without having been able to prove it, that a stronger version of sub/superharmonicity holds also in dimension $n \geq 3$ (a possibility would be that $\left(1-E_{\Omega}\right)^{(n-2) / n}$ is subharmonic).

\section{Riesz Potentials AND THE EXPONENTIAL TRANSFORM}

We start by reviewing some properties of the Riesz kernels [21], [22], [25], here denoted $K_{\alpha}$ or $I_{\alpha}$ depending on which normalization is chosen. For $\alpha=2$ they produce the Newtonian potentials while for the critical index value $\alpha=0$, after a "renormalization" procedure consisting of subtraction of the singular term and taking an exponential, they lead to what we call the exponential transform.

The most straight-forward definition of the Riesz kernel is

$$
K_{\alpha}(x)=\frac{1}{|x|^{n-\alpha}} .
$$

It is a locally integrable function in $\mathbb{R}^{n}$ whenever the parameter $\alpha$ is a complex number with $\operatorname{Re} \alpha>0$. In particular, $K_{\alpha}$ is a distribution in $\mathbb{R}^{n}$ for these $\alpha$. It is well-known that the map $\alpha \mapsto K_{\alpha}$ is analytic regarded as a map from the right half-plane into the Fréchet space of distributions and that it, as such a map, has a meromorphic extension to the entire complex plane, with simple poles at $\alpha=0$, $-2,-4, \ldots$ as the only singularities.

For example, the meromorphic extension to $\operatorname{Re} \alpha>-2$ is given as follows. For any $\varphi \in C_{0}^{\infty}\left(\mathbb{R}^{n}\right)$ and $R>0$ we can write, for $\operatorname{Re} \alpha>0$,

$$
\begin{aligned}
\left\langle K_{\alpha}, \varphi\right\rangle & =\int \frac{\varphi(x) d x}{|x|^{n-\alpha}} \\
& =\int_{|x|<R} \frac{\varphi(x)-\varphi(0)}{|x|^{n-\alpha}} d x+\int_{|x| \geq R} \frac{\varphi(x) d x}{|x|^{n-\alpha}}+\frac{\left|S^{n-1}\right| R^{\alpha}}{\alpha} \varphi(0) .
\end{aligned}
$$


Here

$$
\frac{\left|S^{n-1}\right| R^{\alpha}}{\alpha}=\int_{|x|<R} \frac{d x}{|x|^{n-\alpha}},
$$

and $\left|S^{n-1}\right|=2 \pi^{n / 2} / \Gamma(n / 2)$ denotes the area of the unit sphere.

Choosing $R=1$ for simplicity, the last term in (2.1) is the action of the distribution $\left(\left|S^{n-1}\right| / \alpha\right) \delta$ on $\varphi, \delta$ denoting the Dirac measure at the origin. As a function of $\alpha$, that distribution is meromorphic in the entire complex plane, with a pole only at $\alpha=0$.

The middle term in (2.1) is well-defined as a distribution acting on $\varphi$ for all $\alpha \in \mathbb{C}$, whereas the first term is well-defined for $\operatorname{Re} \alpha>-1$; in fact even for $\operatorname{Re} \alpha>-2$, by interpreting the integral as a principal value integral. In summary we can write, for $\operatorname{Re} \alpha>0$,

$$
K_{\alpha}=L_{\alpha}+\frac{\left|S^{n-1}\right|}{\alpha} \delta
$$

where the distribution $L_{\alpha}$ is defined whenever $\operatorname{Re} \alpha>-2$. It is readily verified that $L_{\alpha}$ is analytic in $\alpha$, so (2.2) defines the desired meromorphic continuation of $K_{\alpha}$. We may think of $L_{\alpha}$ as the regular part of $K_{\alpha}$ near $\alpha=0$.

A different way of regularizing $K_{\alpha}$ at $\alpha=0$ is to multiply it with $\alpha$ : the map $\alpha \mapsto \alpha K_{\alpha}$ is regular analytic for all $\operatorname{Re} \alpha>-2$, and by (2.2) we can recover $L_{0}$ from it as

$$
L_{0}=\left.\frac{d}{d \alpha}\right|_{\alpha=0}\left(\alpha K_{\alpha}\right)
$$

Similarly, if we want to regularize $K_{\alpha}$ at all poles $\alpha=0,-2,-4, \ldots$ we may divide it with the gamma function $\Gamma(\alpha / 2)$, which has poles at the same points and has no zeros. Thus $K_{\alpha} / \Gamma(\alpha / 2)$ is analytic everywhere. This brings us one step closer to the traditional definition of the Riesz kernel, which is

$$
I_{\alpha}(x)=\frac{\Gamma\left(\frac{n-\alpha}{2}\right)}{2^{\alpha} \pi^{n / 2} \Gamma(\alpha / 2)} K_{\alpha}(x) .
$$

Here one has introduced new poles at $\alpha=n, n+2, n+4, \ldots$, coming from the factor $\Gamma((n-\alpha) / 2)$. The definition of $I_{\alpha}$ is adapted so that the Fourier transform becomes simple:

$$
\hat{I}_{\alpha}(\xi)=\frac{1}{|\xi|^{\alpha}}
$$

where $\hat{f}(\xi)=\int f(x) e^{-i x \cdot \xi} d x$, generally speaking. It follows that, e.g.,

$$
-\Delta I_{\alpha}=I_{\alpha-2}, \quad I_{\alpha} * I_{\beta}=I_{\alpha+\beta}, \quad I_{0}=\delta,
$$

whenever the index values $n, n+2, n+4, \ldots$ do not occur. 
If $\mu$ is a Radon measure with compact support (or otherwise such that the convolution make sense) we set

$$
I_{\alpha}^{\mu}=I_{\alpha} * \mu .
$$

Similarly, $K_{\alpha}^{\mu}=K_{\alpha} * \mu, L_{\alpha}^{\mu}=L_{\alpha} * \mu$. Also, we denote by $U^{\mu}$ the usual Newtonian potential of $\mu$ normalized so that $-\Delta U^{\mu}=\mu$, namely

$$
\begin{array}{ll}
U^{\mu}(x)=\frac{1}{2 \pi} \int \log \frac{1}{|x-y|} d \mu(y) & (n=2), \\
U^{\mu}(x)=\frac{1}{(n-2)\left|S^{n-1}\right|} \int \frac{d \mu(y)}{|x-y|^{n-2}} \quad(n \geq 3) .
\end{array}
$$

In both cases the gradient is given by

$$
\nabla U^{\mu}(x)=\frac{1}{\left|S^{n-1}\right|} \int \frac{x-y}{|x-y|^{n}} d \mu(y) \quad(n \geq 2) .
$$

From the properties of $I_{\alpha}$ above, we see that $U^{\mu}=I_{2}^{\mu}$ when $n \geq 3$. When $n=2, I_{2}^{\mu}$ does not exist, but $U^{\mu}$ can still be obtained in various ways from $I_{\alpha}^{\mu}$ (or $\left.K_{\alpha}^{\mu}\right)$ as $\alpha \rightarrow 2$, e.g.,

$$
U^{\mu}=\left.\frac{1}{2 \pi} \frac{d}{d \alpha}\right|_{\alpha=2} K_{\alpha}^{\mu} .
$$

Let us now return to the decomposition (2.2), which convoluted with $\mu$ becomes

$$
K_{\alpha}^{\mu}=L_{\alpha}^{\mu}+\frac{\left|S^{n-1}\right|}{\alpha} \mu .
$$

Here we shall be particularly interested in the regular part $L_{\alpha}^{\mu}$ for the critical index value $\alpha=0$. Moreover, we shall restrict ourselves to absolutely continuous measures $\mu$ of the form

$$
d \mu=\rho d x, \quad 0 \leq \rho \leq 1 .
$$

The density function $\rho$ will be subject also to some further restrictions.

For measures $\mu$ as in (2.6) it turns out that more appropriate than $L_{0}^{\mu}$ itself is the exponential of it, with a certain normalization factor:

$$
E_{\mu}(x)=\exp \left[-\frac{2}{\left|S^{n-1}\right|} L_{0}^{\mu}(x)\right] .
$$

This is our exponential transform. $2 /\left|S^{n-1}\right|$ is adapted to the upper bound for $\rho$ in a sense which will become clear later (see after Lemma 4.1). With the bounds for $\rho$ changed to $0 \leq \rho \leq A$ the constant would be $2 /\left(A\left|S^{n-1}\right|\right)$. 
Since from now on $\mu$ will always be of the form (2.6) we shall write $E_{\rho}$ rather than $E_{\mu}$. Similarly for other expressions ( $L_{0}^{\rho}$ etc.). Assuming first that $\rho$ is smooth, let us spell out the definition of $E_{\rho}$. By (2.1), (2.2) we have, for any $R>0$ and for $\varphi \in C_{0}^{\infty}\left(\mathbb{R}^{n}\right)$,

$$
\left\langle L_{\alpha}, \varphi\right\rangle=\int_{|x|<R} \frac{\varphi(x)-\varphi(0)}{|x|^{n-\alpha}} d x+\int_{|x| \geq R} \frac{\varphi(x) d x}{|x|^{n-\alpha}}+\frac{\left|S^{n-1}\right|\left(R^{\alpha}-1\right)}{\alpha} \varphi(0) .
$$

Hence

$$
\left\langle L_{0}, \varphi\right\rangle=\int_{|x|<R} \frac{\varphi(x)-\varphi(0)}{|x|^{n}} d x+\int_{|x| \geq R} \frac{\varphi(x) d x}{|x|^{n}}+\varphi(0)\left|S^{n-1}\right| \log R,
$$

giving

$$
L_{0}^{\rho}(x)=\int_{|x-y|<R} \frac{\rho(y)-\rho(x)}{|x-y|^{n}} d y+\int_{|x-y| \geq R} \frac{\rho(y) d y}{|x-y|^{n}}+\rho(x)\left|S^{n-1}\right| \log R
$$

and so

$$
\begin{aligned}
E_{\rho}(x)=\frac{1}{R^{2 \rho(x)}} \exp \left[\frac{2}{\left|S^{n-1}\right|} \int_{|x-y|<R} \frac{\rho(x)-\rho(y)}{|x-y|^{n}} d y\right. & \\
& \left.\quad-\frac{2}{\left|S^{n-1}\right|} \int_{|x-y| \geq R} \frac{\rho(y) d y}{|x-y|^{n}}\right] .
\end{aligned}
$$

Letting $R \rightarrow 0$ this simplifies to

$$
E_{\rho}(x)=\lim _{R \rightarrow 0} \frac{1}{R^{2 \rho(x)}} \exp \left[-\frac{2}{\mid S^{n-1 \mid}} \int_{|x-y| \geq R} \frac{\rho(y) d y}{|x-y|^{n}}\right] .
$$

Above we have assumed that $\rho$ is smooth and has compact support. With the convention that $\exp [-\infty]=0$ we can allow also certain nonsmooth densities $\rho$ in (2.8). In order for (2.8) to make sense it is enough to make sure that, for each $x \in \mathbb{R}^{n},(\rho(x)-\rho(y)) /|x-y|^{n}$ is bounded from above by an integrable function of $y$, for $y$ in a neighbourhood of $x$. This is the case for example if $\rho$ is the characteristic function of an open set: $\rho=\chi_{\Omega}, \Omega \subset \mathbb{R}^{n}$ open. (Then $(\rho(x)-\rho(y)) /|x-y|^{n} \leq 0$ for $y$ in a neighbourhood of $x$.) No restriction on the size of $\Omega$ is needed because the worst thing that can happen is that the exponent in (2.8) becomes minus infinity, and in that case we simply have $E_{\rho}(x)=0$. It is also easy to see that (2.9) remains valid when $\rho=\chi_{\Omega}$.

The case that $\rho=\chi_{\Omega}, \Omega \subset \mathbb{R}^{n}$ open, is really our main interest in this paper. In that case $E_{\rho}(x)$ makes a big jump, basically from zero to plus infinity, as $x$ passes from the exterior of $\Omega$ to the interior. Therefore the exterior exponential transform, $\left.E_{\rho}\right|_{\mathbb{R}} n_{\backslash \Omega}$, is in effect disconnected from the interior one, $\left.E_{\rho}\right|_{\Omega}$. In order to conform notation with that of some previous papers (e.g. [10], [11]) and to avoid possible confusion in the two-dimensional case, where another version of 
the exponential transform in the interior exists (see Section 6), we shall make a slight change of notation for the case $\rho=\chi_{\Omega}$ : We write $E_{\Omega}=\left.E_{\rho}\right|_{\mathbb{R}^{n} \backslash \Omega}$ and call it the exterior exponential transform (or simply the "exponential transform"), and we write $H_{\Omega}=\left.E_{\rho}\right|_{\Omega}$ and call it the interior exponential transform (the name "adjoint exponential transform" has also been used [11]).

Since $\rho(x)$ is either one or zero when $\rho=\chi_{\Omega}$ the definitions of $E_{\Omega}$ and $H_{\Omega}$ can be slightly simplified, so let us summarize the definitions as follows.

Definition 2.1. The (exterior) exponential transform of an open set $\Omega \subset \mathbb{R}^{n}$ is

$$
E_{\Omega}(x)=\exp \left[-\frac{2}{\left|S^{n-1}\right|} \int_{\Omega} \frac{d y}{|x-y|^{n}}\right]
$$

defined for $x \in \mathbb{R}^{n} \backslash \Omega$. Whenever convenient we extend $E_{\Omega}$ to all $\mathbb{R}^{n}$ by the same formula (2.10), i.e., by setting $E_{\Omega}(x)=0$ for $x \in \Omega$.

The interior exponential transform of $\Omega$ is, for any $R>0$,

$$
\begin{aligned}
H_{\Omega}(x)=\frac{1}{R^{2}} \exp \left[\frac{2}{\left|S^{n-1}\right|} \int_{B(x, R) \backslash \Omega} \frac{d y}{|x-y|^{n}}\right. & \\
& \left.\quad-\frac{2}{\left|S^{n-1}\right|} \int_{\Omega \backslash B(x, R)} \frac{d y}{|x-y|^{n}}\right]=\frac{E_{\Omega \backslash B(x, R)}(x)}{R^{2} E_{B(x, R) \backslash \Omega}(x)},
\end{aligned}
$$

defined for $x \in \Omega$. If $R>0$ is chosen so small that $B(x, R) \subset \Omega$, the formula simplifies to

$$
H_{\Omega}(x)=\frac{1}{R^{2}} E_{\Omega \backslash B(x, R)}(x),
$$

while if $\Omega$ is bounded and $R>0$ is chosen so large so that $\Omega \subset B(x, R)$

$$
H_{\Omega}(x)=\frac{1}{R^{2} E_{B(x, R) \backslash \Omega}(x)} .
$$

If we let $R \rightarrow \infty$ in (2.13) then we may take the center of the ball independent of $x$ : for any $a \in \mathbb{R}^{n}$

$$
H_{\Omega}(x)=\lim _{R \rightarrow \infty} \frac{1}{R^{2} E_{B(a, R) \backslash \Omega}(x)},
$$

with uniform convergence on compact subsets of $\Omega$. Moreover, $\Omega$ need not be bounded in this formula. Thus the interior exponential transform of a domain may be viewed as one over a renormalized version of the exterior exponential transform of the complementary domain.

Some elementary observations concerning $E_{\Omega}$ and $H_{\Omega}$ are contained in the following proposition. 
Proposition 2.2.

(i) If $\int_{\Omega \backslash B(0,1)} d y /|y|^{n}=\infty$ (which can occur only if $\Omega$ is unbounded), then $E_{\Omega}$ and $H_{\Omega}$ are both identically zero. If $\int_{\Omega \backslash B(0,1)} d y /|y|^{n}<\infty$ and $\Omega$ is nonempty, then $0<E_{\Omega}(x)<1$ for all $x \in \mathbb{R}^{n} \backslash \bar{\Omega}, 0<H_{\Omega}(x)<\infty$ for all $x \in \Omega$.

For $x \in \partial \Omega$ we have $E_{\Omega}(x)=0$ if $\Omega$ is thick enough at $x$, for example has positive Lebesgue density there.

(ii) $E_{\Omega}$ and $H_{\Omega}$ are monotone decreasing with respect to $\Omega$ : If $\Omega_{1} \subset \Omega_{2}$, then

$$
\begin{aligned}
& E_{\Omega_{1}}(x) \geq E_{\Omega_{2}}(x) \quad\left(x \in \mathbb{R}^{n} \backslash \Omega_{2}\right), \\
& H_{\Omega_{1}}(x) \geq H_{\Omega_{2}}(x) \quad\left(x \in \Omega_{1}\right) \text {. }
\end{aligned}
$$

(iii) $E_{\rho}$ is multiplicative in $\rho: E_{\rho_{1}+\rho_{2}}=E_{\rho_{1}} E_{\rho_{2}}$. It follows for example that if $\Omega_{1}$ and $\Omega_{2}$ are disjoint and $x \in \Omega_{1}$, then

$$
H_{\Omega_{1} \cup \Omega_{2}}(x)=H_{\Omega_{1}}(x) E_{\Omega_{2}}(x) .
$$

(iv) $E_{\Omega}(x)$ increases as $x$ moves away from the convex hull of $\Omega$. Precisely, if for example $\Omega \subset\left\{x \in \mathbb{R}^{n} \mid x_{n}<0\right\}$, then $\left(\partial E_{\Omega} / \partial x_{n}\right)(x) \geq 0$ when $x_{n}>0$.

(v) $E_{\Omega}(x)$ and $H_{\Omega}(x)$ behave under affine conformal maps as

$$
E_{t A \Omega+a}(t A x+a)=E_{\Omega}(x),
$$

and

$$
H_{t A \Omega+a}(t A x+a)=\frac{1}{t^{2}} H_{\Omega}(x) .
$$

Here $t>0, A \in O(n), a \in \mathbb{R}^{n}$.

(vi) The germ of $E_{\rho}$ at infinity (or at any point outside supp $\rho$ ) determines $\rho$ completely. ments.

Proof. Most statements are immediate from the definitions. Just a few com-

(i) The last statement is made more precise (and proved) in Lemma 5.1 below.

(ii) The inequality (2.16) is most easily seen from (2.12). Despite (2.15), (2.16) $E_{\rho}$ (defined by (2.8)) is not monotone decreasing in $\rho$ in general. Equivalently, $L_{0}$ is not a positive distribution.

(iv) This is obvious since, referring to the definition (2.10) of $E_{\Omega}(x), x$ only occurs in the factor $|x-y|$ which for each fixed $y \in \Omega$ increases as $x$ moves away from the convex hull of $\Omega$, and $E_{\Omega}(x)$ itself is increasing in that factor.

(vi) This is a special case of the following general fact concerning Riesz potentials: a measure $\mu$ is completely determined by the germ of $K_{\alpha}^{\mu}$ at any point outside $\operatorname{supp} \mu$, except in the cases $\alpha=2,4,6, \ldots$ and $\alpha=n, n+2, n+4$, .... See [21], [22]. Note that the Newtonian potential belongs to the exceptional cases $(\alpha=2)$. The idea behind the proof of the above statement is 
that knowing the germ at a point implies knowing all the derivatives at the same point, and the derivatives at e.g. infinity of $K_{\alpha}^{\mu}$ determine, as a simple computation shows, all the moments $\int x_{1}^{\gamma_{1}} \cdots x_{n}^{\gamma_{n}} d \mu(x)\left(\gamma_{j} \geq 0\right.$ integers $)$ in the case of a nonexceptional value of $\alpha$.

Statement (vi) in the proposition indicates that $E_{\rho}$ could be a useful object in studying moment problems and other related inverse problems. This is indeed the case, at least in dimension two [19], [20].

\section{BOUNDARY INTEGRAL FORMULAS FOR THE EXPONENTIAL TRANSFORMS}

In this section we shall find boundary integral formulas for the exponential transforms when $\rho$ is of the form $\rho=\chi_{\Omega}$, with $\Omega$ a bounded domain with smooth boundary. We also write up the corresponding formulas for the ordinary potentials, which we then denote $U^{\Omega}, K_{\alpha}^{\Omega}, L_{\alpha}^{\Omega}, I_{\alpha}^{\Omega}$ (in place of $U^{\rho}$ etc.).

We shall use the formalism of exterior differential forms and the Hodge star operator [7], [26]. The latter is defined on basic forms

$$
\omega=d x_{i_{1}} \wedge \cdots \wedge d x_{i_{p}} \quad \text { as } \quad * \omega= \pm d x_{i_{p+1}} \wedge \cdots \wedge d x_{i_{n}},
$$

where the sign is chosen so that

$$
\omega \wedge * \omega=d x_{1} \wedge \cdots \wedge d x_{n}=d x \quad \text { (the volume form) }
$$

and $\left(i_{1}, \ldots, i_{n}\right)$ denotes any permutation of $(1, \ldots, n)$.

Setting $r=|x|$ we define the "solid angle form" $d \theta$ by

$$
d \theta=\frac{*(r d r)}{r^{n}}=\frac{*\left(x_{1} d x_{1}+\cdots+x_{n} d x_{n}\right)}{|x|^{n}} .
$$

This is an $(n-1)$-form defined in $\mathbb{R}^{n} \backslash\{0\}$. It can be integrated over (measurable subsets of) any oriented (n-1)-dimensional submanifold of $\mathbb{R}^{n} \backslash\{0\}$. For us, this submanifold will usually be given as $\partial \Omega$ for some domain $\Omega \subset \mathbb{R}^{n}$, and then the orientation will be the natural orientation as a boundary (so that Stokes' formula holds). The unit sphere $S^{n-1}$ will always be oriented as the boundary of $B(0,1)$, and if $A$ is a subset of $S^{n-1}$ then $\int_{A} d \theta$ has the interpretation of being the solid angle occupied by $A$, or simply the hypersurface measure of $A$.

Despite the notation, $d \theta$ is not exact, only locally exact, i.e., closed: $d(d \theta)=$ 0 . We still prefer the notation $d \theta$ because we feel it is suggestive. For example, in two dimensions $d \theta$ reduces to the usual thing, namely the differential of the angle variable defined locally by $\theta=\arctan \left(x_{2} / x_{1}\right)$ when $x_{1} \neq 0$. 
Some useful relations are

$$
\begin{aligned}
d r \wedge * d r & =d x_{1} \wedge \cdots \wedge d x_{n}=d x \\
* d(\log r) & =d \theta \quad(n=2), \\
* d\left(r^{2-n}\right) & =(2-n) d \theta \quad(n \geq 3) .
\end{aligned}
$$

Note that the latter two equations relate $d \theta$ to the Newtonian potential. We refer to [7] (in particular Sections 6.1 and 7.1) for an elegant treatment of the above matters.

If $f$ is a smooth function in $\mathbb{R}^{n}$ we have, outside the origin,

$$
\begin{aligned}
d(f d \theta)=d f \wedge d \theta & =\sum_{i=1}^{n} \frac{\partial f}{\partial x_{i}} d x_{i} \wedge \frac{*\left(x_{1} d x_{1}+\cdots+x_{n} d x_{n}\right)}{|x|^{n}} \\
& =\sum_{i=1}^{n} \frac{\partial f}{\partial x_{i}} \frac{x_{i} d x_{1} \wedge \cdots \wedge d x_{n}}{|x|^{n}}=\frac{x}{|x|^{n}} \cdot \nabla f(x) d x .
\end{aligned}
$$

Thus, if $\Omega$ is a smoothly bounded domain with $0 \notin \bar{\Omega}$, Stokes' formula gives

$$
\int_{\partial \Omega} f(x) d \theta(x)=\int_{\Omega} \frac{x}{|x|^{n}} \cdot \nabla f(x) d x .
$$

If $0 \in \Omega$ we get instead

$$
\int_{\partial \Omega} f(x) d \theta(x)-\left|S^{n-1}\right| f(0)=\int_{\Omega} \frac{x}{|x|^{n}} \cdot \nabla f(x) d x,
$$

as is seen by applying (3.1) to $\Omega \backslash B(0, \varepsilon)$ and letting $\varepsilon \rightarrow 0$.

Now, applying (3.1) with $f(x)=|x|^{\alpha}(\alpha \in \mathbb{R}), f(x)=|x|^{\alpha}(\log |x|-1 / \alpha)$ $(\alpha \neq 0), f(x)=x|x|^{\alpha}$ (vector-valued) and $f(x)=\log |x|$ gives, respectively,

$$
\begin{aligned}
& \int_{\partial \Omega}|x|^{\alpha} d \theta(x)=\alpha \int_{\Omega} \frac{d x}{|x|^{n-\alpha}}, \\
& \int_{\partial \Omega}|x|^{\alpha}\left(\log |x|-\frac{1}{\alpha}\right) d \theta(x)=\alpha \int_{\Omega} \frac{\log |x|}{|x|^{n-\alpha}} d x \quad(\alpha \neq 0), \\
& \int_{\partial \Omega} x|x|^{\alpha} d \theta(x)=(\alpha+1) \int_{\Omega} \frac{x}{|x|^{n-\alpha}} d x, \\
& \int_{\partial \Omega} \log |x| d \theta(x)=\int_{\Omega} \frac{d x}{|x|^{n}}
\end{aligned}
$$

if $0 \notin \bar{\Omega}$. By (3.2) we may allow also $0 \in \Omega$ in the first three formulas, provided $\alpha>0$. As a substitute for the last formula when $0 \in \Omega$ we have

$$
\int_{\partial \Omega} \log |x| d \theta(x)-\left|S^{n-1}\right| \log \varepsilon=\int_{\Omega \backslash B(0, \varepsilon)} \frac{d x}{|x|^{n}},
$$


where $\varepsilon>0$ is chosen so that $B(0, \varepsilon) \subset \Omega$.

Changing variable of integration and moving the origin to an arbitrary point, which we call $x$, the above formulas give the following expressions for our potential theoretic quantities.

Proposition 3.1. If $\Omega \subset \mathbb{R}^{n}$ is bounded and $\partial \Omega$ is smooth (or piecewise smooth), then

$$
\begin{aligned}
& U^{\Omega}(x)=\frac{1}{8 \pi} \int_{\partial \Omega}|x-y|^{2}\left(1+2 \log \frac{1}{|x-y|}\right) d \theta(y-x) \quad(n=2, x \notin \partial \Omega), \\
& U^{\Omega}(x)=\frac{1}{2(n-2)\left|S^{n-1}\right|} \int_{\partial \Omega}|x-y|^{2} d \theta(y-x) \quad(n \geq 3, x \notin \partial \Omega), \\
& \nabla U^{\Omega}(x)=\frac{1}{\left|S^{n-1}\right|} \int_{\partial \Omega}(x-y) d \theta(y-x) \quad(n \geq 2, x \notin \partial \Omega), \\
& K_{\alpha}^{\Omega}(x)=\frac{1}{\alpha} \int_{\partial \Omega}|x-y|^{\alpha} d \theta(y-x) \quad(n \geq 2, x \notin \partial \Omega), \\
& L_{0}^{\Omega}(x)=\int_{\partial \Omega} \log |x-y| d \theta(y-x) \quad(n \geq 2, x \notin \partial \Omega), \\
& E_{\Omega}(x)=\exp \left[-\frac{2}{\left|S^{n-1}\right|} \int_{\partial \Omega} \log |x-y| d \theta(y-x)\right] \quad(n \geq 2, x \notin \bar{\Omega}), \\
& H_{\Omega}(x)=\exp \left[-\frac{2}{\left|S^{n-1}\right|} \int_{\partial \Omega} \log |x-y| d \theta(y-x)\right] \quad(n \geq 2, x \in \Omega) .
\end{aligned}
$$

Note that the expressions for $E_{\Omega}(x)$ and $H_{\Omega}(x)$ are exactly the same. The only potentials lacking above are $L_{\alpha}^{\Omega}$ for $\alpha \neq 0$ and $I_{\alpha}^{\Omega}$, but these are obtained directly from $K_{\alpha}^{\Omega}$, e.g., $L_{\alpha}^{\Omega}(x)=K_{\alpha}^{\Omega}(x)-\left(\left|S^{n-1}\right| / \alpha\right) \chi_{\Omega}(x)$.

If $\Omega$ is starshaped with respect to $x \in \Omega$, then $d \theta(y-x)$ can be regarded as a positive measure (in the variable $y$ ) on $\partial \Omega$ of total mass $\left|S^{n-1}\right|$. Thus (3.9) shows that $H_{\Omega}(x)$ can be interpreted as the geometric mean of $1 /|x-y|^{2}$ over $y \in \partial \Omega$ with respect to $d \theta(y-x)$ in this case. Similarly, the other quantities above can be considered as suitable geometric or arithmetic meanvalues. For example, $-\nabla U^{\Omega}(x)$ for $x \in \Omega$ is by (3.5) the arithmetic mean of the radius vector from $x$ to points $y$ on $\partial \Omega$. Note however that if $\Omega$ is not starshaped with respect to $x$, then $d \theta(y-x)$ is not positive, and if $x$ is outside $\Omega$ then $d \theta(y-x)$ even has zero net mass.

The exponential transforms can also be expressed in terms of spherical means. Let for any $x \in \mathbb{R}^{n}, r>0$,

$$
A(r ; x)=\frac{|\partial B(x, r) \cap \Omega|}{|\partial B(x, r)|}
$$

be the fraction of the sphere of radius $r$ around $x$ which is inside $\Omega$. 
Since

$$
\begin{aligned}
\int_{\Omega} \frac{d y}{|x-y|^{n-\alpha}} & =\left|S^{n-1}\right| \int_{0}^{\infty} A(r ; x) r^{\alpha-1} d r \\
& = \begin{cases}-\left|S^{n-1}\right| \int_{0}^{\infty} \log r d A(r ; x) & \text { if } \alpha=0, \\
-\frac{\left|S^{n-1}\right|}{\alpha} \int_{0}^{\infty} r^{\alpha} d A(r ; x) & \text { if } \alpha \neq 0\end{cases}
\end{aligned}
$$

we have

$$
\begin{aligned}
& E_{\Omega}(x)=\exp \left[-2 \int_{0}^{\infty} A(r ; x) \frac{d r}{r}\right]=\exp \left[2 \int_{0}^{\infty} \log r d A(r ; x)\right], \\
& H_{\Omega}(x)=\frac{1}{R} \exp \left[-2 \int_{R}^{\infty} A(r ; x) \frac{d r}{r}\right]=\exp \left[2 \int_{0}^{\infty} \log r d A(r ; x)\right],
\end{aligned}
$$

where $R>0$ is any number smaller than (or equal to) the distance from $x \in \Omega$ to the boundary.

Similarly we have, for the Newtonian potential,

$$
U^{\Omega}(x)=\frac{1}{n-2} \int_{0}^{\infty} A(r ; x) r d r=-\frac{1}{2(n-2)} \int_{0}^{\infty} r^{2} d A(r ; x)
$$

if $n \geq 3$ and

$$
U^{\Omega}(x)=-\int_{0}^{\infty} A(r ; x) r \log r d r=-\frac{1}{4} \int_{0}^{\infty} r^{2}(2 \log r-1) d A(r ; x)
$$

if $n=2$.

These formulas again exhibit the exponential transform as a geometric mean in cases in which the ordinary Newtonian potential appear as an arithmetic mean. For example, when $x \in \Omega, n \geq 3$ and $\Omega$ is starshaped with respect to $x, H_{\Omega}(x)$ is one over the geometric mean of $r^{2}$ with respect to the probability measure (in $r)-d A(r ; x)$ and $U^{\Omega}(x)$ is a constant factor times the corresponding arithmetic mean.

Put in a more general perspective we may define, for any real number $p \neq 0$,

$$
M_{p}(x)=\left[-\int_{0}^{\infty} r^{2 p} d A(r ; x)\right]^{1 / p}
$$

and

$$
M_{0}(x)=\lim _{p \rightarrow 0} M_{p}(x)=\exp \left[-\int_{0}^{\infty} \log r^{2} d A(r ; x)\right]
$$


Then $M_{1}$ is the arithmetic, $M_{0}$ the geometric and $M_{-1}$ the harmonic mean value of $r^{2}$ with respect to $-d A(r ; x)$ and, when $n \geq 3$,

$$
U^{\Omega}(x)=\frac{1}{2(n-2)} M_{1}(x), \quad H_{\Omega}(x)=\frac{1}{M_{0}(x)} .
$$

Similar formulas hold for the boundary integrals in Proposition 3.1.

\section{EXAMPLES}

We shall compute $E_{\Omega}$ for balls and infinite plates, and $H_{\Omega}$ for any domain bounded by a quadratic surface.

A. Domains bounded by planes. From (3.8) and (3.9) we see that $E_{\Omega}$ and $H_{\Omega}$ for plates can be derived from the following formula, which gives the jump of the integral in (3.8), (3.9) as $x$ crosses a hyperplane.

Lemma 4.1. Let $L=\left\{x \in \mathbb{R}^{n} \mid x_{n}=0\right\}$, oriented as the boundary of the half space $\left\{x_{n}<0\right\}$. Then

$$
\exp \left[-\frac{2}{\left|S^{n-1}\right|} \int_{L} \log |x-y| d \theta(y-x)\right]= \begin{cases}a_{n} x_{n} & \text { if } x_{n}>0, \\ -\left(a_{n} x_{n}\right)^{-1} & \text { if } x_{n}<0,\end{cases}
$$

where the constant $a_{n}$ is given by

$$
a_{n}=\exp \left[\frac{\int_{0}^{\pi / 2} \sin ^{n-2} \theta \log \frac{1}{\cos \theta} d \theta}{\int_{0}^{\pi / 2} \sin ^{n-2} \theta d \theta}\right] .
$$

We have, e.g., $a_{2}=2, a_{3}=e$.

Proof. Note that if $x_{n}>0$, the exponential to be computed can be interpreted as the geometric mean of the distance from $x$ to points $y$ on $L$ with respect to the probability measure (in $y)-\left(2 /\left|S^{n-1}\right|\right) d \theta(y-x)$ on $L$. It follows that it must equal some constant (called $a_{n}$ ) times $\left|x_{n}\right|$. Thus the first case of the formula is proved, apart from the calculation of $a_{n}$, which we omit.

For $x_{n}<0$ we get $1 / a_{n}\left|x_{n}\right|$ instead because of the opposite sign of $d \theta(y-x)$ when $L$ is seen from the other side.

Note that Lemma 4.1 and its proof clarifies the role of the constant $2 /\left|S^{n-1}\right|$ in the definition of the exponential transform: it is exactly that constant which gives a linear decay of $E_{\Omega}$ at $\partial \Omega$ in the case that $\partial \Omega$ is flat, or smooth. Similarly, $H_{\Omega}$ tends to infinity as one over the distance at smooth parts of the boundary.

Next we extend the homogeneity argument implicit in the proof of Proposi- Author: Proposition 3.1, tion 3.1 from flat boundaries to cones. 
Lemma 4.2. Let $\Omega \subset \mathbb{R}^{n}$ be a cone with vertex at the origin (which means that $t \Omega=\Omega$ for all $t>0)$ and with $\partial \Omega \backslash\{0\}$ smooth. Define $0<\alpha<1$ by

$$
\alpha=\frac{\left|S^{n-1} \cap \Omega\right|}{\left|S^{n-1}\right|} .
$$

Then there exists a continuous function $g$ on $S^{n-1}$ such that $g>0$ in $S^{n-1} \backslash \partial \Omega$, $g=0$ on $S^{n-1} \cap \partial \Omega$ and such that, in terms of polar coordinates $x=r \omega(r>0$, $\left.\omega \in S^{n-1}\right)$ on $\mathbb{R}^{n} \backslash\{0\}$,

(4.1) $\exp \left[-\frac{2}{\left|S^{n-1}\right|} \int_{\partial \Omega} \log |x-y| d \theta(y-x)\right]= \begin{cases}g(\omega) r^{2 \alpha} & \text { if } x \notin \bar{\Omega}, \\ g(\omega)^{-1} r^{2 \alpha-2} & \text { if } x \in \Omega .\end{cases}$

(Note however that $E_{\Omega}$ and $H_{\Omega}$ themselves are identically zero due to the size of $\Omega$ at infinity.)

Proof. We first observe that, for any $x \in \Omega$,

$$
\int_{\partial \Omega} d \theta(y-x)=\left|S^{n-1} \backslash \bar{\Omega}\right|=(1-\alpha)\left|S^{n-1}\right| .
$$

This is because for each direction $\omega \in \Omega \cap S^{n-1}$, the ray $\{x+t \omega \mid t>0\}$ will eventually (for $t$ large) stay in $\Omega$, hence if it passes through $\partial \Omega$ at all it will do this an even number of times, giving pairwise cancelling contributions to the integral (4.2). Thus such directions do not contribute to the integral in (4.2).

For similar reasons, every direction $\omega \in S^{n-1} \backslash \bar{\Omega}$ gives the same net contribution to the integral as if there is exactly one point $y \in \partial \Omega$ in this direction. Thus the total contribution from such directions is $\left|S^{n-1} \backslash \bar{\Omega}\right|$. Now (4.2) follows.

For $x \notin \bar{\Omega}$ we get instead

$$
\int_{\partial \Omega} d \theta(y-x)=-\left|\Omega \cap S^{n-1}\right|=-\alpha\left|S^{n-1}\right|,
$$

the roles of $\Omega \cap S^{n-1}$ and $S^{n-1} \backslash \bar{\Omega}$ being interchanged and the minus sign coming from the fact that $x$ is now on the other side of the oriented surface $\partial \Omega$. This finishes the proof of (4.2).

Now we turn to the main statement of the lemma, which is a question of homogeneity. Multiplying $x$ in the left member of (4.1) by a factor $t>0$ gives, 
assuming $x \in \Omega$ for example,

$$
\begin{aligned}
\exp [ & \left.-\frac{2}{\left|S^{n-1}\right|} \int_{\partial \Omega} \log |t x-y| d \theta(y-t x)\right] \\
& =\exp \left[-\frac{2}{\left|S^{n-1}\right|} \int_{\partial \Omega} \log |t x-t y| d \theta(t y-t x)\right] \\
& =\exp \left[-\frac{2}{\left|S^{n-1}\right|} \int_{\partial \Omega}(\log t+\log |x-y|) d \theta(y-x)\right] \\
& =\exp \left[-\frac{2}{\left|S^{n-1}\right|}\left(\left|S^{n-1}\right| \bar{\Omega}\left|\log t+\int_{\partial \Omega} \log \right| x-y \mid d \theta(y-x)\right)\right] \\
& =t^{2 \alpha-2} \exp \left[-\frac{2}{\left|S^{n-1}\right|} \int_{\partial \Omega} \log |x-y| d \theta(y-x)\right] .
\end{aligned}
$$

From this the case $x \in \Omega$ in (4.1) follows. The case $x \notin \bar{\Omega}$ is similar.

Finally, the statements about $g(\omega)$ follow by restricting to $r=1$ and using that $\partial \Omega$ is smooth. (The boundary behaviour at $\partial \Omega$ will be the same as for the exponential transform and its adjoint for a truncated version of $\Omega$, for example $\Omega \cap B(0,2)$.

From Lemma 4.1 we immediately get the exponential transforms for plates.

Corollary 4.3. Let $\Omega$ be the domain bounded by two parallel planes $L_{1}$ and $L_{2}$ and let $d_{j}(x)$ denote the distance from a point $x \in \mathbb{R}^{n}$ to $L_{j}$. Then

$$
E_{\Omega}(x)=\left(\frac{d_{1}(x)}{d_{2}(x)}\right)^{ \pm 1} \quad(x \notin \Omega),
$$

where the sign of the exponent is chosen so that the index of the nearest plane is in the numerator (giving $E_{\Omega}(x)<1$ ), and

$$
H_{\Omega}(x)=\frac{1}{a_{n}^{2} d_{1}(x) d_{2}(x)} \quad(x \in \Omega) .
$$

Proof. Equations (3.8), (3.9) were stated merely for bounded domains, so we have to exhaust $\Omega$ by such domains, e.g. by $\Omega \cap B(0, R), R \rightarrow \infty$. Since $\int_{\Omega \cap \partial B(0, R)} \log |x-y| d \theta(y-x) \rightarrow 0$ as $R \rightarrow \infty$, application of Lemma 4.1 to each component of $\partial \Omega$ then gives the corollary, taking into account that $x$ is on different sides of the two oriented components of $\partial \Omega$ when $x \notin \Omega$, on the same side when $x \in \Omega$.

The second formula, (4.4), can also be viewed as an instance of Theorem 4.4 below. 


\section{B. The interior exponential transform for domains bounded by quadratic surfaces.}

Theorem 4.4. Let $\Omega=\left\{x \in \mathbb{R}^{n} \mid q(x)<0\right\}$, where $q(x)$ is any polynomial of degree two in $x_{1}, \ldots, x_{n}$ with positive semidefinite quadratic part $q_{2}(x)$. Then

$$
H_{\Omega}(x)=-\frac{C}{q(x)},
$$

where the constant $C \geq 0$ equals the geometric mean of $q_{2}$ over $S^{n-1}$ :

$$
C=\exp \left[\frac{1}{\left|S^{n-1}\right|} \int_{S^{n-1}} \log q_{2}(\omega) d \theta(\omega)\right] .
$$

Proof. We shall use the boundary integral formula (3.9) for the interior transform. Fix $x \in \Omega$ and parametrize $\partial \Omega$ by the angle (direction) $\omega \in S^{n-1}$ from $x$ to $y \in \partial \Omega$.

With the possible exception of a nullset of directions $\omega \in S^{n-1}$, the straight line with direction $\omega$ and infinitely extended in both directions intersects $\partial \Omega$ exactly twice. Therefore it is enough to use only, for example, the upper hemisphere $S_{+}^{n-1}$ as a parameter space for $\omega$.

Let $y_{1}(\omega)$ and $y_{2}(\omega)$ denote the two points where the straight line with direction $\omega \in S_{+}^{n-1}$ intersects $\partial \Omega$. Then $y_{j}(\omega)=x+t_{j}(\omega) \omega$ where $t=t_{j}(\omega)$, $j=1,2$, are the solutions of

$$
q(x+t \omega)=0 .
$$

This is a second degree equation in $t$, say $q(x+t \omega)=a_{0}+a_{1} t+a_{2} t^{2}$, where the coefficients $a_{j}$ depend on $x$ and $\omega$. It is easy to see, however, that $a_{0}$ depends only on $x$ and $a_{2}$ only on $\omega$. Indeed, $a_{0}=q(x), a_{2}=q_{2}(\omega)$.

Using that the product of the two solutions of (4.7) equals $a_{0} / a_{2}$ we therefore get

$$
\begin{aligned}
H_{\Omega}(x) & =\exp \left[-\frac{2}{\left|S^{n-1}\right|} \int_{\partial \Omega} \log |x-y| d \theta(y-x)\right] \\
& =\exp \left[-\frac{1}{\left|S_{+}^{n-1}\right|} \int_{S_{+}^{n-1}}\left(\log \left|t_{1}(\omega)\right|+\log \left|t_{2}(\omega)\right|\right) d \theta(\omega)\right] \\
& =\exp \left[-\frac{1}{\left|S_{+}^{n-1}\right|} \int_{S_{+}^{n-1}} \log \left|\frac{q(x)}{q_{2}(\omega)}\right| d \theta(\omega)\right] \\
& =\frac{C}{|q(x)|}=-\frac{C}{q(x)},
\end{aligned}
$$

with $C$ as in the statement of the theorem. This completes the proof. 
C. The exponential transform for balls. In view of (2.18) it is enough to treat the unit ball $\Omega=B_{n}=B(0,1) \subset \mathbb{R}^{n}$. Clearly the exponential transform of this depends only on $r=|x|$, and we shall write $E_{n}(r)$ in place of $E_{B n}(x)$.

Theorem 4.5. We have, for $r>1$,

$$
E_{1}(r)=\frac{r-1}{r+1}, \quad E_{2}(r)=1-\frac{1}{r^{2}}
$$

and, inductively,

$$
E_{n}(r)=E_{n-2}(r) \exp \left[\frac{2}{(n-2) r^{n-2}}\right]
$$

for $n \geq 3$.

Continuing the list, we thus get

$$
\begin{aligned}
& E_{3}(r)=\frac{r-1}{r+1} \exp \left[\frac{2}{r}\right], \\
& E_{4}(r)=\left(1-\frac{1}{r^{2}}\right) \exp \left[\frac{1}{r^{2}}\right], \\
& E_{5}(r)=\frac{r-1}{r+1} \exp \left[\frac{2}{r}+\frac{2}{3 r^{3}}\right], \\
& E_{6}(r)=\left(1-\frac{1}{r^{2}}\right) \exp \left[\frac{1}{r^{2}}+\frac{1}{2 r^{4}}\right],
\end{aligned}
$$

etcetera.

Remark 4.6. Since we only are interested in dimensions $n \geq 2$ in this paper, the first value $n=1$ above can be thought of as just a way to start the induction process. On the other hand, most of our results actually make sense and are valid also for $n=1$, the formulas for $n \geq 3$ then being in force for that case.

Proof. The formula for $E_{1}(r)$ can be viewed as a special case of (4.3), and the formula for $E_{2}(r)$ has been computed in [19], [10], for example.

For the recursion formula we may take $x=(0, \ldots, 0, r), r>1$. Then, passing to polar coordinates in dimension $n-1$ by setting $y^{\prime}=\left(y_{1}, \ldots, y_{n-1}\right)=$ $\rho \omega, \omega \in S^{n-2}, t=y_{n}$ so that $d y=d \omega \rho^{n-2} d \rho d t$, we have

$$
\begin{aligned}
\int_{B_{n}} \frac{d y}{|x-y|^{n}} & =\int_{|y|<1} \frac{d y_{1} \cdots d y_{n}}{\left(y_{1}^{2}+\cdots+y_{n-1}^{2}+\left(y_{n}-r\right)^{2}\right)^{n / 2}} \\
& =\int_{-1}^{1} d t \int_{0}^{\sqrt{1-t^{2}}} \int_{S^{n-2}} \frac{d \omega \rho^{n-2} d \rho}{\left(\rho^{2}+(r-t)^{2}\right)^{n / 2}} \\
& =\left|S^{n-2}\right| \int_{-1}^{1} I_{n}\left(\frac{\sqrt{1-t^{2}}}{r-t}\right) \frac{d t}{r-t} .
\end{aligned}
$$


Here we have set

$$
I_{n}(s)=\int_{0}^{s} \frac{\sigma^{n-2} d \sigma}{\left(1+\sigma^{2}\right)^{n / 2}} .
$$

Partial integration in the definition of $I_{n}(s)$ gives the recursion formula

$$
(n-2) I_{n}(s)=(n-3) I_{n-2}(s)-\frac{s^{n-3}}{\left(1+s^{2}\right)^{(n-2) / 2}},
$$

valid for $n \geq 3$. Inserting this into the previous formula gives

$$
\begin{aligned}
\frac{2}{\left|S^{n-1}\right|} \int_{B_{n}} \frac{d y}{|x-y|^{n}}=\frac{2}{\left|S^{n-3}\right|} \int_{B_{n-2}} & \frac{d y}{|x-y|^{n-2}} \\
& -\frac{2\left|S^{n-2}\right|}{(n-2)\left|S^{n-1}\right|} \int_{-1}^{1} \frac{\left(1-t^{2}\right)^{(n-3) / 2} d t}{\left(r^{2}-2 r t+1\right)^{(n-2) / 2}} .
\end{aligned}
$$

Substituting $t=\cos \varphi, 0 \leq \varphi \leq \pi$, in the last integral yields

$$
\int_{-1}^{1} \frac{\left(1-t^{2}\right)^{(n-3) / 2} d t}{\left(r^{2}-2 r t+1\right)^{(n-2) / 2}}=\frac{1}{r^{n-2}} \int_{0}^{\pi}\left(\frac{\sin ^{2} \varphi}{1-2 r^{-1} \cos \varphi+r^{-2}}\right)^{(n-2) / 2} d \varphi .
$$

Quite surprisingly, the integral in the right member turns out not to depend on $r$. This is an instance of (setting $t=r^{-1}$ ).

Lemma 4.7. For any smooth function $\Phi$ of one variable we have

$$
\int_{0}^{\pi} \Phi\left(\frac{\sin ^{2} \varphi}{1-2 t \cos \varphi+t^{2}}\right) d \varphi=\int_{0}^{\pi} \Phi\left(\sin ^{2} \varphi\right) d \varphi
$$

for $0 \leq t<1$.

Assuming the lemma proved and using that $\left|S^{n-1}\right|=\left|S^{n-2}\right| \int_{0}^{\pi} \sin ^{n-2} \varphi d \varphi$, we get

$$
\begin{aligned}
& \frac{2\left|S^{n-2}\right|}{(n-2)\left|S^{n-1}\right|} \int_{-1}^{1} \frac{\left(1-t^{2}\right)^{(n-3) / 2} d t}{\left(r^{2}-2 r t+1\right)^{(n-2) / 2}} \\
& \quad=\frac{2\left|S^{n-2}\right|}{(n-2)\left|S^{n-1}\right|} \frac{1}{r^{n-2}} \int_{0}^{\pi} \sin ^{n-2} \varphi d \varphi=\frac{2}{(n-2) r^{n-2}} .
\end{aligned}
$$

Inserting this into the previous formulas and taking the exponential then gives the desired recursion formula (4.8).

A proof of the lemma can be found in the appendix of [16]. Let us still try to explain the result by a purely geometric argument. What the lemma says is that the functions of $\varphi$,

$$
f_{t}(\varphi)=\frac{\sin ^{2} \varphi}{1-2 t \cos \varphi+t^{2}},
$$


all have the same distribution function, i.e., that the function (of $\lambda$ )

$$
M_{t}(\lambda)=\operatorname{meas}\left\{\varphi \in(0, \pi) \mid f_{t}(\varphi)<\lambda\right\}
$$

is the same for all values of the parameter $t$. It is enough to consider values of $\lambda$ in the range of $f_{t}$, namely the interval $(0,1]$.

Consider the point $(\cos \varphi, \sin \varphi)$ on the upper half $S_{+}^{1}$ of the unit circle in $\mathbb{R}^{2}$. The distance from this point to $(t, 0)$ is

$$
\sqrt{1-2 t \cos \varphi+t^{2}}
$$

and the distance to its projection $(\cos \varphi, 0)$ onto the $x_{1}$-axis is $\sin \varphi$. It follows that, denoting by $\alpha=\alpha_{t}(\varphi), 0<\alpha<\pi$, the angle at $(t, 0)$ between the positive $x$-axis and the ray to $(\cos \varphi, \sin \varphi)$, we have

$$
\sin \alpha=\frac{\sin \varphi}{\sqrt{1-2 t \cos \varphi+t^{2}}}=\sqrt{f_{t}(\varphi)} .
$$

From this we see that the set of points $(\cos \varphi, \sin \varphi)$ on $S_{+}^{1}$ for which $f_{t}(\varphi)<\lambda$ consists of the two segments which are delimited from above by the two rays from $(t, 0)$ with angles $\alpha=\arcsin \sqrt{\lambda}$ and $\alpha=\pi-\arcsin \sqrt{\lambda}$, respectively, and delimited from below by the $x$-axis.

By a reflection of the leftmost of these segments in the $x$-axis we get the alternate description of $M_{t}(\lambda)$ as the total length of the two segments on the full cirle $S^{1}$ which are squeezed between the $x$-axis and the line (extended in both directions) through $(t, 0)$ making the angle arcsin $\sqrt{\lambda}$ with the $x$-axis.

Now we start increasing $t$, keeping $\lambda$ fixed. Then the the above mentioned line moves in a parallel fashion to the right. But one can also view this as a parallel motion in the normal direction of the line (indeed, the component of the motion along the line has no effect). From this point of view it is obvious that $M_{t}(\lambda)$ remains constant: of the two segments, the one in the upper semicircle decreases with exactly the same speed as the one in the lower semicircle decreases, simply because the two points of intersection between the line and the circle move with equal velocity.

This finishes the proof of Lemma 4.7 and hence of Theorem 4.5.

D. Shell domains. Let $\Omega=B(0, R) \backslash \overline{B(0, r)}$ where $0<r<R$. By (2.18), Theorems 4.4 and 4.5 we have (using the notation of Theorem 4.5)

$$
E_{B(0, R)}(x)=E_{n}\left(\frac{|x|}{R}\right), \quad H_{B(0, R)}=\frac{1}{R^{2}-|x|^{2}}
$$

and similarly with $r$ in place of $R$. This gives 


$$
\begin{aligned}
& E_{\Omega}(x)=\frac{E_{B(0, R)}(x)}{E_{B(0, r)}(x)}=\frac{E_{n}(|x| / R)}{E_{n}(|x| / r)} \quad(|x|>R), \\
& H_{\Omega}(x)=\frac{H_{B(0, R)}(x)}{E_{B(0, r)}(x)}=\frac{1}{E_{n}(|x| / r)\left(R^{2}-|x|^{2}\right)} \quad(r<|x|<R), \\
& E_{\Omega}(x)=\frac{H_{B(0, R)}(x)}{H_{B(0, r)}(x)}=\frac{r^{2}-|x|^{2}}{R^{2}-|x|^{2}} \quad(|x|<r) .
\end{aligned}
$$

E. Cylinders. Let $\Omega \subset \mathbb{R}^{n}$ and let $m \geq 1$. For the cylinder $\Omega \times \mathbb{R}^{m}$ in $\mathbb{R}^{n+m}$ we have

$$
\begin{aligned}
E_{\Omega \times \mathbb{R}^{m}}(x, \xi) & =E_{\Omega}(x) & & \left(x \in \mathbb{R}^{n} \backslash \Omega, \xi \in \mathbb{R}^{m}\right), \\
H_{\Omega \times \mathbb{R}^{m}}(x, \xi) & =c_{n m} H_{\Omega}(x) & & \left(x \in \Omega, \xi \in \mathbb{R}^{m}\right)
\end{aligned}
$$

for a certain constant $0<c_{n m}<1$.

The proof for $E_{\Omega \times \mathbb{R}} m$ is a direct calculation: one simply performs the integration over $\mathbb{R}^{m}$ in the integral defining $E_{\Omega \times \mathbb{R}^{m}}(x, \xi)$.

For $H_{\Omega \times \mathbb{R}} m(x, \xi)$ one may subtract a suitable ball, say $B=B(x, R) \subset \Omega$, and then use (2.17) and (4.5) together with the formula for $E_{\Omega \times \mathbb{R}^{m}}$. Since a defining function for $B \times \mathbb{R}^{m}$ is $q(x, \xi)=x_{1}^{2}+\cdots+x_{n}^{2}-R^{2}$, one gets

$$
\begin{aligned}
& H_{\Omega \times \mathbb{R}} m(x, \xi)=H_{B \times \mathbb{R}} m(x, \xi) E_{(\Omega \backslash B) \times \mathbb{R}} m(x, \xi) \\
& =H_{B \times \mathbb{R}} m(x, \xi) E_{\Omega \backslash B}(x) \\
& =\frac{c_{n m}}{R^{2}-\left(x_{1}^{2}+\cdots+x_{n}^{2}\right)} E_{\Omega \backslash B}(x) \\
& =c_{n m} H_{B}(x) E_{\Omega \backslash B}(x)=c_{n m} H_{\Omega}(x) \text {, }
\end{aligned}
$$

where

$$
c_{n m}=\exp \left[\frac{1}{\left|S^{n+m-1}\right|} \int_{S^{n+m-1}} \log \left(\theta_{1}^{2}+\cdots+\theta_{n}^{2}\right) d \theta\right] \text { by (4.6). }
$$

\section{Estimates AND BOUNDARY BEHAVOIUR}

Let $\Omega \subset \mathbb{R}^{n}$ be open. In this section it is natural to have $E_{\Omega}$ extended by zero in $\Omega$ (cf. Definition 2.1). We first study the continuity of $E=E_{\Omega}$ up to the boundary.

Lemma 5.1. Set

$$
Z=\left\{x \in \partial \Omega \mid \int_{\Omega \cap B(x, 1)} \frac{d y}{|x-y|^{n}}<+\infty\right\} .
$$


Then $E$ is continuous in $\mathbb{R}^{n} \backslash Z$ and $E(x)=0$ for all $x \in \bar{\Omega} \backslash Z$. If $E$ is not identically zero, then it is discontinuous at every point of $Z$ and $E(x)>0$ for every $x \in Z \cup\left(\mathbb{R}^{n} \backslash \bar{\Omega}\right)$.

The set $Z$ is an exceptional subset of $\partial \Omega$ in the sense that $\Omega$ has Lebesgue density zero at each point of $Z$. Also, $\partial \Omega \backslash Z$ is dense in $\partial \Omega$.

Proof. We may assume that $E$ is not identically zero. It is obvious that $E$ is continuous at all points of $\mathbb{R}^{n} \backslash \partial \Omega$. Since $E=0$ in $\Omega, E$ can be continuous at a point $x \in \partial \Omega$ only if $E(x)=0$, i.e., only if $x \notin Z$.

On the other hand, if $x \in \partial \Omega \backslash Z$ then Fatou's lemma gives, for any sequence $x_{k} \rightarrow x\left(x_{k} \in \mathbb{R}^{n}\right)$,

$$
+\infty=\int_{\Omega} \frac{d y}{|x-y|^{n}} \leq \varliminf_{k \rightarrow \infty} \int_{\Omega} \frac{d y}{\left|x_{k}-y\right|^{n}},
$$

showing that $E\left(x_{k}\right) \rightarrow E(x)$. Thus $E(x)$ is continuous at each $x \in \partial \Omega \backslash Z$.

If $x \in Z$, then

$$
0 \leq \frac{1}{\varepsilon^{n}} \int_{\Omega \cap B(x, \varepsilon)} d y \leq \int_{\Omega \cap B(x, \varepsilon)} \frac{d y}{|x-y|^{n}} \rightarrow 0
$$

as $\varepsilon \rightarrow 0$, showing that $\Omega$ has vanishing Lebesgue density at $x$. The density of $\partial \Omega \backslash Z$ in $\partial \Omega$ is an easy exercise (or see after (2.11) in [10]). This finishes the proof.

Remark 5.2. Polarizing $E(x)$ into a function of two independent variables $x, y \in \mathbb{R}^{n}$ by

$$
E(x, y)=\exp \left[-\frac{2}{\mid S^{n-1 \mid}} \int_{\Omega} \frac{d \xi}{|x-\xi|^{\alpha}|y-\xi|^{\beta}}\right],
$$

where $0<\alpha, \beta<n, \alpha+\beta=n$, one can show that $E(x, y)$ always is continuous in $x$ and $y$ separately. The proof is a straight-forward generalization of a corresponding proof concerning the complex analytic polarization (see Section 6) of $E$ in two dimensions. See [10] for that proof.

A slight improvement to Lemma 5.1 is the following result.

Theorem 5.3. Assume that $\Omega$ satisfies an inner ball condition, namely that there exists $r>0$ such that for every $y \in \partial \Omega$ one can find $a \in \Omega$ with $B(a, r) \subset \Omega$, $y \in \partial B(a, r)$. Then $E$ is Lipschitz continuous. More precisely, there exists a constant $C_{n}<\infty$ depending only on dimension $n$ such that, for all $x, y \in \mathbb{R}^{n}$ and with $r>0$ as above,

$$
|E(x)-E(y)| \leq \frac{C_{n}}{r}|x-y| .
$$


In particular,

$$
E(x) \leq \frac{C_{n}}{r} d(x)
$$

$d(x)$ denoting the distance from $x$ to $\Omega$.

Proof. By Lemma 5.1, $E(x)=0$ for all $x \in \bar{\Omega}$. We first estimate the gradient of $E(x)$ outside $\bar{\Omega}$. Let $x \in \mathbb{R}^{n} \backslash \bar{\Omega}$, let $z$ be a nearest point on $\partial \Omega$ (so that $|x-z|=d(x))$ and let $a \in \Omega$ be the center of a ball of radius $r$ as in the statement of the inner ball condition. Then using monotonicity (2.15), invariance under scaling (2.18), that $|x-a|=r+d(x)$, and the fact that the exponential transforms $E_{n}(r)$ for balls (see Theorem 4.5) satisfy $E_{n}(1+t) \leq c_{n} t(t>0)$ some for some constant $c_{n}$ (in fact, $c_{n}=E_{n}^{\prime}(1)$ works) we get

$$
\begin{aligned}
|\nabla E(x)| & \leq \frac{2 n}{\left|S^{n-1}\right|} \int_{\Omega} \frac{d y}{|x-y|^{n+1}} E_{\Omega}(x) \\
& \leq \frac{2 n}{\left|S^{n-1}\right|} \int_{|y-x|>d(x)} \frac{d y}{|x-y|^{n+1}} E_{B(a, r)}(x) \\
& =2 n \int_{\rho>d(x)} \frac{d \rho}{\rho^{2}} E_{B(0, r)}(r+d(x)) \leq \frac{2 n}{d(x)} E_{n}\left(1+\frac{d(x)}{r}\right) \\
& \leq \frac{2 n c_{n}}{r} .
\end{aligned}
$$

From this we conclude that (5.1) holds (with $C_{n}=2 n c_{n}$ ) whenever the full line segment between $x$ and $y$ stays in the complement of $\bar{\Omega}$.

With the same notations as above we also estimate

$$
\begin{aligned}
E(x) & \leq E_{B(a, r)}(x)=E_{B(0, r)}(|x-a|) \\
& =E_{B(0, r)}(r+|x-z|) \\
& =E_{n}\left(1+\frac{|x-z|}{r}\right) \\
& \leq c_{n} \frac{|x-z|}{r} .
\end{aligned}
$$

Thus, (5.2) holds, and combining this with the previous estimate we also get (5.1) for arbitrary points $x, y \in \mathbb{R}^{n}$. This completes the proof.

In the opposite direction we have that $E(x)$ always lifts at least quadratically with the distance from the boundary (which by (4.1) is best possible without additional assumptions), and lifts linearly if a strong form of outer ball condition holds (such a condition is satisfied for example if $\Omega$ is convex or if $\partial \Omega$ is smooth of class $C^{2}$ ). More precisely, we have the following result. 


\section{Theorem 5.4.}

(i) Suppose $\Omega$ is bounded with diameter $\operatorname{diam}(\Omega)$. Then

$$
E(x) \geq\left(\frac{d(x)}{d(x)+\operatorname{diam}(\Omega)}\right)^{2} .
$$

(ii) Suppose $\Omega$ is bounded and there exists $r>0$ such that for every $x \notin \bar{\Omega}$ with $d(x)<r$ there is a ball $B(a, r) \subset \mathbb{R}^{n} \backslash \Omega$ such that $d(x)+|x-a|=r$ (i.e., such that $x \in B(a, r)$ and the radius from $a$ through $x$ hits $\partial B(a, r)$ at a point belonging to $\partial \Omega$ ). Then

$$
E(x) \geq \frac{r d(x)}{(r+\operatorname{diam}(\Omega))^{2}}
$$

for $x \in \mathbb{R}^{n}$ with $d(x)<r$.

Remark 5.5. In the convex case we also get the estimate

$$
E(x) \geq \frac{d(x)}{d(x)+\operatorname{diam}(\Omega)},
$$

by squeezing between two hyperplanes and using (4.3).

Proof. Let $x \in \mathbb{R}^{n} \backslash \bar{\Omega}$. Without any further assumptions we may use that $\Omega$ is contained in the shell domain $D=B(x, R) \backslash \overline{B(x, r)}$, where $r=d(x)$ and $R=d(x)+\operatorname{diam}(\Omega)$. By (2.15) and (4.11) we then get

$$
E_{\Omega}(x) \geq E_{D}(x)=\frac{r^{2}}{R^{2}},
$$

showing the estimate in part (i) of the lemma.

As for the estimate in part (ii) we change the above shell domain to $D=$ $B(a, R) \backslash \overline{B(a, r)}$ with $r$ and $a$ as in the statement and $R=r+\operatorname{diam}(\Omega)$. This gives, assuming $a=0$ for simplicity of notation,

$$
\begin{aligned}
E_{\Omega}(x) \geq E_{D}(x) & =\frac{r^{2}-|x|^{2}}{R^{2}-|x|^{2}} \\
& =\frac{(r-|x|)(r+|x|)}{\left(R^{2}-|x|^{2}\right)} \geq \frac{d(x) r}{R^{2}} \\
& =\frac{r d(x)}{(r+\operatorname{diam}(\Omega))^{2}},
\end{aligned}
$$

as desired. 
With similar proofs we obtain corresponding estimates for $H=H_{\Omega}$.

Theorem 5.6. Let $\delta(x)$ denote the distance from $x \in \Omega$ to $\mathbb{R}^{n} \backslash \Omega$.

(i) Without any further assumptions we have

$$
H(x) \leq \frac{1}{\delta(x)^{2}} .
$$

Author: Theorem 5.3, right?

(ii) If $\Omega$ satisfies the inner ball condition in Theorem 5.3, we have

$$
H(x) \leq \frac{1}{r \delta(x)} .
$$

(iii) Suppose $\Omega$ is bounded and satisfies a strong form of outer ball condition (similar to that in (ii) of Theorem 5.4), namely that there exists $r>0$ such that for every $x \in \Omega$ there exists a ball $B(a, r) \subset \mathbb{R}^{n} \backslash \Omega$ with $\delta(x)+r=|x-a|$. Then, with the same $C_{n}$ as in Theorem 5.3,

$$
H(x) \geq \frac{r}{C_{n} \delta(x)(r+\operatorname{diam}(\Omega))^{2}} .
$$

The above estimates hold for all $x \in \Omega$.

As to higher regularity a natural question is whether $\partial \Omega$ being smooth to some degree implies that $\left.E\right|_{\Omega^{c}}$ is correspondingly smooth up to the boundary. In two dimensions one form of positive answer to this question was given in [20]: if $\partial \Omega$ is real analytic, then also $\left.E\right|_{\mathbb{R}}{ }^{n} \backslash \Omega$ is real analytic up to $\partial \Omega$ (i.e., $\left.E\right|_{\mathbb{R}^{n} \backslash \Omega}$ has a real analytic continuation across $\partial \Omega$ ). In [10] this statement was pushed a bit further: $E_{\Omega}$ has a real analytic extension across $\partial \Omega$ if and only if the exterior Newtonian field $\nabla U^{\Omega}$ (or, equivalently, the Cauchy transform of $\Omega$ ) has such an extension.

The proofs of these two-dimensional results use in a crucial way a special feature available in two dimension, namely that the exponential transform in a natural way polarizes into a function of two complex variables. This will be the topic of the next section.

For the higher dimensional case we have so far only partial results (not to be presented in this paper) concerning analytic continuation across an analytic boundary.

\section{ON THE TWO-DIMENSIONAL EXPONENTIAL TRANSFORM AND ANALYTIC CONTINUATION}

In two dimensions, with $\mathbb{R}^{2}$ identified with the complex plane $\mathbb{C}$ in the usual way, the exponential transform of a domain $\Omega$ has previously [19], [10], [17] been defined in polarized form as

$$
E_{\Omega}(z, w)=\exp \left[-\frac{1}{\pi} \int_{\Omega} \frac{d A(\zeta)}{(\zeta-z)(\bar{\zeta}-\bar{w})}\right]
$$


for $(z, w) \in \mathbb{C}^{2}, d A$ denoting the Lebesgue measure in two dimensions (area measure). More precisely, (6.1) is the polarized version of our exterior exponential transform. Note that $E_{\Omega}(z, w)$ is well-defined and nontrivial also inside $\Omega$. Outside $\bar{\Omega}$ it is analytic in $z$, antianalytic in $w$.

The polarized version of the interior exponential transform turns out to be

$$
H_{\Omega}(z, w)=\frac{E_{\Omega}(z, w)}{|z-w|^{2}},
$$

defined at first for $z, w \in \Omega, z \neq w$. If $z$ and $w$ are close to each other in $\Omega$, say if $w \in B(z, R) \subset \Omega$, then using that

$$
E_{B(z, R)}(z, w)=\frac{|z-w|^{2}}{R^{2}}
$$

(see [10]) we can write

$$
H_{\Omega}(z, w)=\frac{E_{B(z, R)}(z, w) E_{\Omega \backslash B(z, R)}(z, w)}{|z-w|^{2}}=R^{-2} E_{\Omega \backslash B(z, R)}(z, w) .
$$

This formula shows that $H_{\Omega}(z, w)$ extends as an antianalytic function in $w$ to a full neighbourhood of $z$. By symmetry it follows that $H_{\Omega}(z, w)$ extends to be analytic in $z$, antianalytic in $w$ in all of $\Omega \times \Omega$. Finally, it is easy to check (see [10]) that

$$
H_{\Omega}(z, w)=-\frac{\partial^{2} E_{\Omega}(z, w)}{\partial \bar{z} \partial w}
$$

$(z, w \in \Omega)$.

Taking $z=w$ in (6.1), (6.3) and comparing with (2.10), (2.12) we see that we can identify the exterior and interior exponential transforms (given by Definition 2.1 ) as the traces on the diagonal of the above polarized transforms: setting $z=$ $x_{1}+i x_{2} \in \mathbb{C}$ for $x=\left(x_{1}, x_{2}\right) \in \mathbb{R}^{2}$ we have

$$
\begin{array}{ll}
E_{\Omega}(x)=E_{\Omega}(z, z) & \left(x \in \mathbb{R}^{2} \backslash \Omega\right), \\
H_{\Omega}(x)=H_{\Omega}(z, z) & (x \in \Omega) .
\end{array}
$$

From now on we suppress $\Omega$ in the notation for the exponential transform (the domain will be kept fixed). It is immediate from $(6.1)$ that if $E(z, w)$ is not identically zero, it tends to one as $|z|$ or $|w|$ tends to infinity. Therefore the Cauchy integral formula applied twice to $1-E(z, w)$ shows, together with (6.4), that

$$
1-E(z, w)=\frac{1}{\pi^{2}} \int_{\Omega} \int_{\Omega} H(u, v) \frac{d A(u)}{u-z} \frac{d A(v)}{\bar{v}-\bar{w}}
$$


$(z, w \in \mathbb{C})$. Thus $1-E$ is a weighted double Cauchy transform of $\Omega$, with analytic/antianalytic weight $H$.

This formula is a useful starting point for discussing analytic continuation properties of $E$, which will be the main topic for this section. Assume that $\partial \Omega$ is smooth real analytic. This is equivalent to saying that there exists a function $S(z)$, the Schwarz function of $\partial \Omega$ [6], [2], [24] defined and analytic in a neighbourhood of $\partial \Omega$ such that

$$
S(z)=\bar{z} \quad \text { for } z \in \partial \Omega .
$$

The map $z \mapsto \overline{S(z)}$ is the anticonformal reflection in $\partial \Omega$.

Using $S(z)$, we get, by a formal use of Stokes' Theorem in (6.5), that for $z$, $w \in \mathbb{C} \backslash \bar{\Omega}$

$$
\begin{aligned}
1-E(z, w) & =\frac{1}{4 \pi^{2}} \int_{\partial \Omega} \int_{\partial \Omega} H(u, v) \frac{\bar{u} d u}{u-z} \frac{v d \bar{v}}{\bar{v}-\bar{w}} \\
& =\frac{1}{4 \pi^{2}} \int_{\partial \Omega} \int_{\partial \Omega} H(u, v) \frac{S(u) d u}{u-z} \frac{\overline{S(v)} d \bar{v}}{\bar{v}-\bar{w}} .
\end{aligned}
$$

In the last expression the integrand is analytic in $u$, antianalytic in $v$ in some neighbourhood of $\partial \Omega$ in $\Omega$, say in $\Omega \backslash K$, where $K \subset \Omega$ is compact. Thus, we can push the contour of integration down to $K$ :

$$
1-E(z, w)=\frac{1}{4 \pi^{2}} \int_{\partial K} \int_{\partial K} H(u, v) \frac{S(u) d u}{u-z} \frac{\overline{S(v)} d \bar{v}}{\bar{v}-\bar{w}}
$$

for $z, w$ outside $\bar{\Omega}$.

From this it is immediate that $E(z, w)$ has an analytic continuation across $\partial \Omega$ : the function

$$
F(z, w)=1-\frac{1}{4 \pi^{2}} \int_{\partial K} \int_{\partial K} H(u, v) \frac{S(u) d u}{u-z} \frac{\overline{S(v)} d \bar{v}}{\bar{v}-\bar{w}}
$$

is analytic/antianalytic for $z, w \in \mathbb{C} \backslash K$ and agrees with $E(z, w)$ for $z, w \in \mathbb{C} \backslash \Omega$.

Next, the right member of (6.6) can be evaluated to give an even more explicit expression for $F(z, w)$, namely

$$
F(z, w)= \begin{cases}E(z, w) & \text { for } z, w \in \mathbb{C} \backslash \Omega \\ (z-\overline{S(w)})(S(z)-\bar{w}) H(z, w) & \text { for } z, w \in \Omega \backslash K\end{cases}
$$


To prove this, let $z, w \in \Omega \backslash K$ be fixed. Then

$$
\begin{aligned}
F(z, w)= & 1-\frac{1}{4 \pi^{2}} \int_{\partial K} \int_{\partial K} H(u, v) \frac{S(u) d u}{u-z} \frac{\overline{S(v)} d \bar{v}}{\bar{v}-\bar{w}} \\
= & 1-\frac{1}{4 \pi^{2}} \int_{\partial K} \int_{\partial K} H(u, v) \frac{S(u)-S(z)}{u-z} \frac{\overline{S(v)}-\overline{S(w)}}{\bar{v}-\bar{w}} d u d \bar{v} \\
= & 1-\frac{1}{4 \pi^{2}} \int_{\partial \Omega} \int_{\partial \Omega} H(u, v) \frac{S(u)-S(z)}{u-z} \frac{\overline{S(v)}-\overline{S(w)}}{\bar{v}-\bar{w}} d u d \bar{v} \\
= & 1-\frac{1}{4 \pi^{2}} \int_{\partial \Omega} \int_{\partial \Omega} H(u, v) \frac{\bar{u}-S(z)}{u-z} \frac{v-\overline{S(w)}}{\bar{v}-\bar{w}} d u d \bar{v} \\
= & 1-\frac{1}{4 \pi^{2}} \int_{\partial \Omega} \int_{\partial \Omega} H(u, v) \frac{\bar{u}}{u-z} \frac{v}{\bar{v}-\bar{w}} d u d \bar{v} \\
& +\frac{1}{4 \pi^{2}} \int_{\partial \Omega} \int_{\partial \Omega} H(u, v) \frac{\bar{u}}{u-z} \frac{\overline{S(w)}}{\bar{v}-\bar{w}} d u d \bar{v} \\
& +\frac{1}{4 \pi^{2}} \int_{\partial \Omega} \int_{\partial \Omega} H(u, v) \frac{S(z)}{u-z} \frac{v}{\bar{v}-\bar{w}} d u d \bar{v} \\
& -\frac{1}{4 \pi^{2}} \int_{\partial \Omega} \int_{\partial \Omega} H(u, v) \frac{S(z)}{u-z} \frac{\overline{S(w)}}{\bar{v}-\bar{w}} d u d \bar{v} .
\end{aligned}
$$

Using (6.2) and the behaviour of $E(z, w)$ at infinity, we have

$$
\begin{aligned}
& \frac{1}{2 \pi i} \int_{\partial \Omega} H(u, v) \frac{\bar{u} d u}{u-z} \\
& \quad=\frac{1}{2 \pi i} \int_{\partial \Omega} \frac{E(u, v) \bar{u} d u}{(u-v)(\bar{u}-\bar{v})(u-z)} \\
& \quad=\frac{1}{2 \pi i} \int_{\partial \Omega} \frac{E(u, v) d u}{(u-v)(u-z)}+\frac{1}{2 \pi i} \int_{\partial \Omega} \frac{E(u, v) \bar{v} d u}{(u-v)(\bar{u}-\bar{v})(u-z)} \\
& \quad=-\frac{1}{2 \pi i} \int_{\partial(\mathbb{C} \mid \Omega)} \frac{E(u, v) d u}{(u-v)(u-z)}+\frac{\bar{v}}{2 \pi i} \int_{\partial \Omega} \frac{H(u, v) d u}{u-z}=\bar{v} H(z, v) .
\end{aligned}
$$

Similarly,

$$
\frac{1}{2 \pi i} \int_{\partial \Omega} H(u, v) \frac{v d \bar{v}}{\bar{v}-\bar{w}}=-u H(u, w)
$$

and

$$
-\frac{1}{4 \pi^{2}} \int_{\partial \Omega} \int_{\partial \Omega} H(u, v) \frac{\bar{u}}{u-z} \frac{v}{\bar{v}-\bar{w}} d u d \bar{v}=-1-z \bar{w} H(z, w) .
$$


Thus,

$$
\begin{aligned}
& F(z, w) \\
& =1+(-1-z \bar{w} H(z, w))+\bar{w} \overline{S(w)} H(z, w)+z S(z) H(z, w)-S(z) \overline{S(w)} H(z, w) \\
& =(z-\overline{S(w)})(S(z)-\bar{w}) H(z, w),
\end{aligned}
$$

proving (6.7) See also [10] for a different derivation of this analytic extension formula.

One advantage with the expression (6.7) for $F(z, w)$ is that it can be specialized to the diagonal $(z=w)$ and then only refers to values of $E$ and $H$ on the diagonal (while setting $z=w$ in (6.6) one still refers to $H(u, v)$ for $u \neq v$ ):

$$
F(z, z)= \begin{cases}E(z, z) & \text { for } z \in \mathbb{C} \backslash \Omega, \\ -|z-\overline{S(z)}|^{2} H(z, z) & \text { for } z \in \Omega \backslash K .\end{cases}
$$

Note the simple geometric interpretation of (6.8): the analytic/antianalytic continuation of $E(z, w)$ at a point $z=w \in \Omega \backslash K$ is obtained by multiplying $H(z, z)$ with minus the square of the distance from $z$ to its reflected point with respect to $\partial \Omega$.

As a curiosity we mention that it is possible, using the boundary integral formulas in Proposition 3.1, to write (6.8) in one stroke as:

$$
F(z, z)=\exp \left[-\frac{1}{\pi} \int_{\partial \Omega} \log \frac{i|u-z|}{|\overline{S(z)}-z|} d \theta(u-z)\right] \quad(z \in \mathbb{C} \backslash K) .
$$

The formula uses that $1 /(2 \pi) \int_{\partial \Omega} d \theta(u-z)$ (integration with respect to $u$ ) equals the characteristic function of $\Omega$.

The formal use of Stokes' theorem in the derivation of (6.6) can really be justified when $\partial \Omega$ is smooth real analytic. Even under the weaker assumption that $\Omega$ is merely a bounded open set having the property that the Cauchy transform of $\chi_{\Omega}$,

$$
\hat{\chi}_{\Omega}(z)=-\frac{1}{\pi} \int_{\Omega} \frac{d A(u)}{u-z},
$$

has an analytic continuation from outside $\Omega$ across $\partial \Omega$, the analytic continuation of $E(z, w)$ as presented above can be justified. That was the main point in [10], and the result was used to prove apriori regularity for boundaries which admit analytic continuation of $\hat{X}_{\Omega}$.

Let us explain this in some more detail. Assume that $\Omega \subset \mathbb{C}$ is just a bounded open set with the property that $\hat{X}_{\Omega}$ has an analytic extension from the exterior of $\Omega$ across $\partial \Omega$. It is convenient, and involves no loss of generality, to assume that the analytic continuation then is presented as the Cauchy transform of something (e.g. a signed measure) with compact support in $\Omega$. Thus we express it as

$$
\hat{\chi}_{\Omega}(z)=\hat{\mu}(z) \quad \text { for } z \in \mathbb{C} \backslash \Omega,
$$


where $\mu$ is a signed measure with $\operatorname{supp} \mu \subset \Omega$. In this case one can define a kind of Schwarz function by

$$
S(z)=\bar{z}-\hat{x}_{\Omega}(z)+\hat{\mu}(z) .
$$

This $S(z)$ is defined everywhere, it is analytic in $\Omega \backslash \operatorname{supp} \mu$, is continuous up to $\partial \Omega$, and equals $\bar{z}$ there. The restriction of $S(z)$ to $\bar{\Omega} \backslash \operatorname{supp} \mu$ can be said to be a one-sided Schwarz function.

For the relationship in general between existence of a one-sided Schwarz function and analyticity of the boundary, see [23]. The result that $E(z, w)$ always admits analytic continuation across a smooth analytic boundary was first obtained in (the first version of) [20].

Now, the main result in [10] states that assuming only (6.9), $E(z, w)$ has an analytic/antianalytic continuation $F(z, w)$ to $(\Omega \backslash K)^{2}$, where $K=\operatorname{supp} \mu$. With $S(z)$ as in (6.10) the continuation is given explicitly by (6.6) or (6.7). Equation (6.6) can here be written as

$$
F(z, w)=1-\frac{1}{\pi^{2}} \int_{K} \int_{K} \frac{H(u, v) d \mu(u) d \overline{\mu(v)}}{(u-z)(\bar{v}-\bar{w})}, \quad(z, w \in \mathbb{C})
$$

which makes $F(z, w)$ defined in all $\mathbb{C} \times \mathbb{C}$ with

$$
-\frac{\partial^{2} F(z, w)}{\partial \bar{z} \partial w}= \begin{cases}\mu(z) \overline{\mu(w)} H(z, w) & \text { in } \Omega \times \Omega, \\ 0 & \text { elsewhere. }\end{cases}
$$

The natural analogue of (6.9) in higher dimensions is

$$
\nabla U^{\Omega}=\nabla U^{\mu} \quad \text { on } \mathbb{R}^{n} \backslash \Omega \text {. }
$$

Writing (6.8) combined with (6.10) in real variable notations it becomes

$$
F(x)= \begin{cases}E(x) & \text { for } x \in \mathbb{R}^{n} \backslash \Omega, \\ -4\left|\nabla U^{\Omega}(x)-\nabla U^{\mu}(x)\right|^{2} H(x) & \text { for } x \in \Omega,\end{cases}
$$

and then makes sense in any number of dimensions. Thus, assuming (6.13), $F$ defined by (6.14) will be real analytic across $\partial \Omega$ when $n=2$. Unfortunately, this statement, even with the constant 4 replaced by any other constant, is false in general when $n \geq 3$. It does hold for polyhedra however (see next section), with the constant 4 replaced by $a_{n}^{2}, a_{n}$ defined in Lemma 4.1 .

In general, returning to two dimensions, equations (6.7), (6.10), (6.11), (6.12) give a relationship between $E, H$ and $\mu$ which sometimes can be made even more explicit and be used to determine one of these quantities when the other two are known. Certainly, $\mu$ is not uniquely determined by $\Omega$, but there are cases in which a natural candidate, which is minimal in some sense, is singled out (a kind of "potential theoretic skeleton" of $\Omega$ ). 
Example 6.1. An illustrative case is the ellipse, for which $H(z, w)$ is known and is of a simple form (by Theorem 3.4) and also a good choice of $\mu$ is known, while $E(z, w)$ is not yet computed (outside $\Omega$ ).

We take the ellipse in standard form:

$$
\Omega=\left\{z=x+i y \in \mathbb{C} \mid \frac{x^{2}}{a^{2}}+\frac{y^{2}}{b^{2}}<1\right\},
$$

where $a>b>0$. Define $c>0$ by $c^{2}=a^{2}-b^{2}$. Writing the equation for $\partial \Omega$ as an equation in $z$ and $\bar{z}$ and solving for $\bar{z}$ gives

$$
\bar{z}=S_{ \pm}(z)=\frac{a^{2}+b^{2}}{c^{2}} z \pm \frac{2 a b}{c^{2}} \sqrt{z^{2}-c^{2}} .
$$

Here $S_{ \pm}(z)$ are the two branches of the algebraic function defined by the equation for $\partial \Omega$. By Theorem 3.4 we then have

$$
H(z, z)=-\frac{C}{\left(\bar{z}-S_{+}(z)\right)\left(\bar{z}-S_{-}(z)\right)}
$$

for some constant $C \geq 0$.

One of the branches $S_{ \pm}(z)$ equals $\bar{z}$ on $\partial \Omega$. If the square root in the expression for $S_{ \pm}$is chosen so that it is positive for large positive values of $z$, the right branch turns out to be $S_{-}(z)$. This hence agrees with the Schwarz function $S(z)$ as defined in (6.10), at least near $\partial \Omega$ in $\Omega$. That definition of $S(z)$ actually presupposes a choice of measure $\mu$. In the present case there is a very natural choice: if we simply make $S_{-}(z)$ single-valued by cutting the complex plane along $[-c, c]$ and then define $S(z)$ as $S_{-}(z)$ in $\Omega$ (considered as a locally integrable function, hence a distribution in $\Omega$ ), then we get $\mu$ from (6.10) as $\mu=\partial S(z) / \partial \bar{z}$. Since taking the $\partial / \partial \bar{z}$ derivative of a function which makes a jump along a line gives half of the size of the jump times arclenght measure along the line we get in the present case that $\mu$ is the measure on $[-c, c]$ given by

$$
d \mu=\frac{2 a b}{c^{2}} \sqrt{c^{2}-x^{2}} d x \quad(x \in[-c, c]) .
$$

Now we insert the above into (6.7). This gives

$$
\begin{aligned}
F(z, z) & =(z-\overline{S(z)})(S(z)-\bar{z}) H(z, z) \\
& =C \frac{|\bar{z}-S(z)|^{2}}{\left(\bar{z}-S_{+}(z)\right)\left(\bar{z}-S_{-}(z)\right)} \\
& =C \frac{z-\overline{S_{-}(z)}}{\bar{z}-S_{+}(z)}
\end{aligned}
$$


The constant $C$ can be evaluated using that $F(z, z)=E(z, z) \rightarrow 1$ as $z \rightarrow \infty$. This gives finally, after polarization (i.e., replacing $\bar{z}$ by $\bar{w}$ ),

$$
F(z, w)=-\frac{a+b}{a-b} \cdot \frac{c^{2} z-\left(a^{2}+b^{2}\right) \bar{w}+2 a b \sqrt{\bar{w}^{2}-c^{2}}}{c^{2} \bar{w}-\left(a^{2}+b^{2}\right) z-2 a b \sqrt{z^{2}-c^{2}}} .
$$

Example 6.2. Another example is when $\Omega$ is a quadrature domain (in the most restricted sense [2]). This means that (6.9) holds with $\mu$ a finite sum of point masses (or derivatives of such):

$$
\mu=\sum_{k=1}^{m} c_{k} \delta_{z_{k}}
$$

where $\delta_{z_{k}}$ denotes the Dirac measure at $z_{k} \in \Omega, c_{k} \in \mathbb{C}$. Repeated occurrence of a point $z_{k}$ may be interpreted as an appropriate derivate of $\delta_{z_{k}}$.

Setting $P(z)=\prod_{k=1}^{m}\left(z-z_{k}\right)$ we have

$$
\hat{\mu}(z)=\frac{R(z)}{P(z)},
$$

where $R(z)$ is a polynomial of degree $m-1$. It was shown in [19] that also $F(z, w)$ is a rational function:

$$
F(z, w)=\frac{Q(z, w)}{P(z) \overline{P(w)}},
$$

where $Q(z, w)$ is a polynomial of degree $m$ in each of $z$ and $\bar{w}$ (total degree $2 m$ ). Restricted to the diagonal $Q$ is a defining function of $\Omega$ :

$$
\Omega=\{z \in \mathbb{C} \mid Q(z, z)<0\}
$$

(up to nullsets). In particular, $\partial \Omega$ is algebraic.

Assuming for simplicity that all the $z_{k}$ are distinct (6.11) becomes

$$
\frac{Q(z, w)}{P(z) \overline{P(w)}}=1-\frac{1}{\pi^{2}} \sum_{k, j=1}^{m} \frac{c_{k} \overline{c_{j}} H\left(z_{k}, z_{j}\right)}{\left(z-z_{k}\right)\left(\bar{w}-\overline{z_{j}}\right)}
$$

thus decomposing the rational function $F(z, w)$ into simple fractions, and (6.12) takes the form

$$
-\pi^{2} \frac{Q\left(z_{k}, z_{j}\right)}{P^{\prime}\left(z_{k}\right) \overline{P^{\prime}\left(z_{j}\right)}}=c_{k} \overline{c_{j}} H\left(z_{k}, z_{j}\right) \quad(k, j=1, \ldots, m) .
$$

A third example of interaction between $E, H$ and $\mu$ is when $\Omega$ is a polyhedron. This is the topic of next section. 


\section{Polyhedra}

One of the few cases in which we have definite results concerning analytic continuation in higher dimensions is when part of $\partial \Omega$ is perfectly flat. In that case we obtain the following, as a consequence of Lemma 4.1.

Proposition 7.1. Assume that $\Omega \subset \mathbb{R}^{n}$ satisfies $0 \in \partial \Omega, \Omega \cap B(0, r)=\{x \in$ $\left.B(0, r) \mid x_{n}<0\right\}$ for some $r>0$. Then the function

$$
F(x)= \begin{cases}E(x) & \text { if } x \in \mathbb{R}^{n} \backslash \Omega, \\ -a_{n}^{2} x_{n}^{2} H(x) & \text { if } x \in \Omega\end{cases}
$$

is real analytic in $\left(\mathbb{R}^{n} \backslash \partial \Omega\right) \cup B(0, r)$. Here $a_{n}$ is the constant in Lemma 4.1.

Proof. We can write

$$
E(x)=\exp \left[-\frac{2}{\left|S^{n-1}\right|} \int_{L} \log |x-y| d \theta(y-x)\right] \cdot G(x)
$$

for $x \in B(0, r) \backslash \Omega$, where $L=\left\{x_{n}=0\right\}=\partial\left\{x_{n}<0\right\}$ and where $G(x)$ is a function which is real analytic in $B(0, r)$. For example, if $\Omega$ is good enough for Proposition 3.1 to be applied we have

$$
G(x)=\exp \left[-\frac{2}{\left|S^{n-1}\right|} \int_{\partial \Omega-L} \log |x-y| d \theta(y-x)\right]
$$

Here $\int_{\partial \Omega-L}$ means $\int_{\partial \Omega}-\int_{L}$.

Similarly we have

$$
H(x)=\exp \left[-\frac{2}{\left|S^{n-1}\right|} \int_{L} \log |x-y| d \theta(y-x)\right] \cdot G(x)
$$

(i.e., the same formula as for $\left.E_{\Omega}(x)\right)$ for $x \in B(0, r) \cap \Omega$. Now Lemma 4.1 shows that

$$
F(x)=a_{n} x_{n} G(x)=-\left(a_{n} x_{n}\right)^{2} H(x)
$$

for $x \in B(0, r) \cap \Omega$, proving the proposition.

ع

Clearly, the ideas of Proposition 7.1 extend to other cases in which we have an explicit analytic continuation of $E(x)$. For example, when part of $\partial \Omega$ is perfectly spherical we can use $\mathbf{B}$ and $\mathbf{C}$ in Section 4.

Applying Proposition 7.1 to polyhedra, say convex polyhedra for simplicity, gives a natural real analytic extension of $E$ down to the "ridge" of the polyhedron. If $\delta(x)$ denotes the distance from $x \in \Omega$ to $\mathbb{R}^{n} \backslash \Omega$, the ridge $R$ of $\Omega$ can be defined as the closure of the set of points $x \in \Omega$ for which $\delta(x)$ is attained for at least two different boundary points. From Proposition 7.1 we easily infer the following. 
Corollary 7.2. Let $\Omega$ be a bounded convex polyhedron. Then the exterior exponential transform $E$ of $\Omega$ has a real analytic continuation $F$ down to the ridge of the polyhedron, explicitly given by

$$
F(x)= \begin{cases}E(x) & \text { for } x \in \mathbb{R}^{n} \backslash \Omega, \\ -a_{n}^{2} \delta(x)^{2} H(x) & \text { for } x \in \Omega \backslash R .\end{cases}
$$

Note the similarity with (6.8). Note also that $F$, as given by the formula, extends to be continuous on all of $\mathbb{R}^{n}$ and that it vanishes (and changes sign) exactly on $\partial \Omega$.

We can also identify a measure $\mu$ as in (6.13). Set

$$
u(x)= \begin{cases}\frac{1}{2} \delta(x)^{2} & \text { in } \Omega, \\ 0 & \text { on } \mathbb{R}^{n} \backslash \Omega .\end{cases}
$$

Then $\Delta u=\chi_{\Omega}-\mu$ for a certain measure $\mu \geq 0$ with support on $R$ (see [9] for more details). It follows that $U^{\mu}=U^{\Omega}+u$, so that

$$
\begin{aligned}
U^{\mu} & =U^{\Omega} & & \text { outside } \Omega, \\
\nabla U^{\mu} & =\nabla U^{\Omega} & & \text { outside } \Omega .
\end{aligned}
$$

We may think of $U^{\mu}$ as the harmonic continuation of $U^{\Omega}$ across $\partial \Omega$ and similarly for the gradients (continuation as a harmonic vector field).

Since

$$
\left|\nabla U^{\mu}-\nabla U^{\Omega}\right|=|\nabla u|=\delta \quad \text { in } \Omega \backslash R,
$$

Corollary 7.2 can be written

$$
F(x)= \begin{cases}E(x) & \text { for } x \in \mathbb{R}^{n} \backslash \Omega, \\ -a_{n}^{2}\left|\nabla U^{\mu}(x)-\nabla U^{\Omega}(x)\right|^{2} H(x) & \text { for } x \in \Omega \backslash R .\end{cases}
$$

When $n=2$ this is the same as (6.14).

In the case of two dimensions we also have the polarized version $F(z, w)$ of $F$ given e.g. by (6.11) (with $K=R$ ). It is analytic/antianalytic in $(\mathbb{C} \backslash R)^{2}$. As $z$ and/or $w$ crosses one of the line segments making up $R$, then $F(z, w)$ makes a jump according to the usual Plemelj formula for the Cauchy integral. For example if both $z$ and $w$ are on $R$, the jump formula is

$$
\begin{array}{r}
\frac{1}{4}[F(z+0, w+0)-F(z+0, w-0)-F(z-0, w+0)+F(z-0, w-0)] n_{z} \overline{n_{w}} \\
=-H(z, w) \lambda(z) \overline{\lambda(w)},
\end{array}
$$


where $\lambda$ denotes the density of $\mu$ with respect to arclength on $R, n(z)$ is the normal vector of $R$ at $z$ and $z \pm 0, w \pm 0$ are used to indicate limits from the two sides of $R(n(z)$ poining to the plus side). This jump formula can also be seen as a spelled out version of (6.12).

Note in particular that $F(z, w)$ by the above is discontinuous on $R$ despite its restriction to the diagonal being continuous (as remarked after Corollary 7.2).

\section{SUB- AND SUPERHARMONICITY}

For $\Omega \subset \mathbb{R}^{n}$ a bounded domain the function $1-E_{\Omega}$ has some resemblance with the equilibrium potential of $\Omega$ (or of its closure). Indeed, $1-E_{\Omega}$ equals one on $\Omega$ and decays to zero at infinity. When $n=2$ it is rather $\frac{1}{2} \log \left(1-E_{\Omega}\right)$ that resembles the equilibrium potential: it is zero on $\Omega$ and decays logarithmically at infinity.

Therefore one naturally asks about sub- and superharmonicity of these functions. In this direction we state the following result.

Theorem 8.1. For any $\Omega \subset \mathbb{R}^{n}$,

$$
\begin{aligned}
& \Delta E_{\Omega} \leq 0 \quad \text { outside } \bar{\Omega}, \\
& \Delta \frac{1}{H_{\Omega}} \leq 0 \quad \text { in } \Omega \text {. }
\end{aligned}
$$

In particular,

$$
\Delta \log H_{\Omega} \geq 0 \quad \text { and (hence) } \quad \Delta H_{\Omega} \geq 0 \quad \text { in } \Omega .
$$

More generally, for any $0 \leq \rho \leq 1$ the exponential transform $E_{\rho}$ (defined by (2.8)) is superharmonic in the interior of the set where $\rho=0$ and subharmonic in the interior of the set where $\rho=1$.

In two dimensions we even have

$$
\Delta \log \left(1-E_{\Omega}\right) \geq 0
$$

(which is stronger than (8.1)), and also

$$
\Delta^{m} E_{\Omega} \leq 0 \text { outside } \bar{\Omega}, \quad \Delta^{m} H_{\Omega} \geq 0 \text { in } \Omega
$$

for all $m=1,2,3, \ldots$

Remark 8.2. We do not know whether (8.4) remains true in higher dimension. However (8.3) fails in general. Indeed, a straightforward computation using Theorem 4.5 shows that $\Delta \log \left(1-E_{\Omega}\right) \leq 0$ in case $\Omega$ is the unit ball in $\mathbb{R}^{3}$ while, by Corollary 4.3, $\Delta \log \left(1-E_{\Omega}\right) \geq 0$ if $\Omega$ is the domain between two parallel planes. Thus $\Delta \log \left(1-E_{\Omega}\right)$ has no definite sign in higher dimensions. One may notice, however, that $\Delta \log E_{\Omega} \leq 0$ always holds, as is seen by a direct calculation. 
We still suspect that something stronger than (8.1) holds in higher dimensions (a possibility would be that $\left.\Delta\left(1-E_{\Omega}\right)^{(n-2) / n} \geq 0\right)$. However, (8.1) is the best we can prove at present.

Proof. We first prove (8.1), or more generally that $E_{\rho}$ is superharmonic outside supp $\rho$. For $x \notin \operatorname{supp} \rho$ we have

$$
\begin{aligned}
E_{\rho}(x) & =\exp \left[-\frac{2}{\left|S^{n-1}\right|} \int \frac{\rho(y) d y}{|x-y|^{n}}\right], \\
\nabla E_{\rho}(x) & =\frac{2 n}{\left|S^{n-1}\right|} \int \frac{(x-y) \rho(y) d y}{|x-y|^{n+2}} \cdot E_{\rho}(x), \\
\Delta E_{\rho}(x) & =\left(\frac{2 n}{\left|S^{n-1}\right|}\right)^{2}\left[\left|\int \frac{(x-y) \rho(y) d y}{|x-y|^{n+2}}\right|^{2}-\frac{\left|S^{n-1}\right|}{n} \int \frac{\rho(y) d y}{|x-y|^{n+2}}\right] E_{\rho}(x) .
\end{aligned}
$$

We may assume that $x=0$, and then the inequality $\Delta E_{\rho}(x) \leq 0$ to be proved becomes

$$
\left|\int \frac{y \rho(y) d y}{|y|^{n+2}}\right|^{2} \leq \frac{\left|S^{n-1}\right|}{n} \int \frac{\rho(y) d y}{|y|^{n+2}} .
$$

Set

$$
A=\int \frac{\rho(y) d y}{|y|^{n+2}} .
$$

Excluding the trivial case $\rho=0$ we have $0<A<\infty$. The left member of (8.5) is the squared length of the vector

$$
\int \frac{y \rho(y) d y}{|y|^{n+2}}
$$

This vector has a certain direction, which we may assume is that of the positive first coordinate axis. Then

$$
\left|\int \frac{y \rho(y) d y}{|y|^{n+2}}\right|=\int \frac{y_{1} \rho(y) d y}{|y|^{n+2}} .
$$

Let $\Omega_{a}=\left\{y \in \mathbb{R}^{n} \mid y_{1}>a\right\}$, with $a>0$ chosen so that

$$
\int_{\Omega_{a}} \frac{d y}{|y|^{n+2}}=A .
$$

Such an $a$ clearly exists (uniquely) since the left member is monotone decreasing in $a$, tending to plus infinity as $a \rightarrow 0$ and tending to zero as $a \rightarrow+\infty$. 
We claim now that $\rho_{a}=\chi_{\Omega_{a}}$ is extremal for (8.5), and hence that it suffices to prove (8.5) for $\rho_{a}$. In fact, comparing our (arbitrary) $\rho$ with $\rho_{a}$ we have

$$
\begin{aligned}
\int \frac{\rho(y) d y}{|y|^{n+2}} & =\int \frac{\rho_{a}(y) d y}{|y|^{n+2}}=A, \\
\left|\int \frac{y \rho(y) d y}{|y|^{n+2}}\right| & =\int \frac{y_{1} \rho(y) d y}{|y|^{n+2}} \\
& \leq \int \frac{y_{1} \rho_{a}(y) d y}{|y|^{n+2}} \\
& =\left|\int \frac{y \rho_{a}(y) d y}{|y|^{n+2}}\right| .
\end{aligned}
$$

Here the inequality comes from the fact that the measure $\rho_{a}(y) d y /|y|^{n+2}$ has the same total mass (namely $A$ ) as $\rho(y) d y /|y|^{n+2}$ and is distributed so as to load $y_{1}$ as heavily as possible under the restriction $0 \leq \rho \leq 1$.

From (8.6), (8.7) it is clear that we only have to verify (8.5) for $\rho=\rho_{a}$. That verification is of course just a straight-forward calculation. However, we can also rely on previously performed computations as follows.

Setting $\Omega_{a, b}=\left\{x \in \mathbb{R}^{n} \mid a<x_{1}<b\right\}$, it is enough to prove (8.5) for all $\rho_{a, b}=\chi_{\Omega_{a, b}}$ and letting $b \rightarrow+\infty$. But for $\rho=\rho_{a, b}$ (8.5) is equivalent to the statement that $\Delta E_{\Omega_{a, b}} \leq 0$ at $x=0$. By Corollary 4.3 we have

$$
E_{\Omega_{a, b}}(x)=\frac{a-x_{1}}{b-x_{1}}
$$

$\left(x_{1}<a\right)$, which indeed is superharmonic for $x_{1}<a$. This proves (8.5), and so (8.1).

Next, (8.2) follows from (8.1) together with (2.14) written on the form

$$
\frac{1}{H_{\Omega}(x)}=\lim _{R \rightarrow \infty} R^{2} E_{B(0, R) \backslash \Omega}(x) .
$$

Here we have uniform convergence on compacts.

The two-dimensional statements (8.4), (8.3) are in principle well-known and consequences of the fact that the polarized interior exponential transform $H_{\Omega}(z, w)$ is a positive semidefinite kernel [5], [17]. Defining

$$
\langle\varphi, \psi\rangle=\frac{1}{\pi^{2}} \int_{\Omega} \int_{\Omega} H(u, v) \varphi(u) \overline{\psi(v)} d A(u) d A(v)
$$

the positive semidefiniteness means that $\langle\varphi, \varphi\rangle \geq 0$ for any $\varphi$ for which $\langle\varphi, \varphi\rangle$ is defined. Thus $\langle\cdot, \cdot\rangle$ is an inner product on a suitable space. In terms of that 
inner product equation (6.5) takes the form

$$
1-E_{\Omega}(z, w)=\left\langle k_{z}, k_{w}\right\rangle, \quad \text { where } k_{z}(u)=\frac{1}{u-z} .
$$

Now (8.3) and (8.4) follow easily:

$$
-\Delta^{m} E(z, z)=\left(4 \frac{\partial^{2}}{\partial \bar{z} \partial z}\right)^{m}(1-E(z, z))=4^{m}\left\langle\frac{\partial^{m} k_{z}}{\partial z^{m}}, \frac{\partial^{m} k_{z}}{\partial z^{m}}\right\rangle \geq 0
$$

for $z \notin \bar{\Omega}$ and, using also the Cauchy-Schwarz inequality for $\langle\cdot, \cdot\rangle$,

$$
\begin{aligned}
\Delta \log (1-E)(z, z) & =4 \frac{\partial^{2}}{\partial \bar{z} \partial z} \log (1-E(z, z)) \\
& =\frac{4}{\left|\left\langle k_{z}, k_{z}\right\rangle\right|^{2}}\left[\left\langle k_{z}, k_{z}\right\rangle\left\langle\frac{\partial k_{z}}{\partial z}, \frac{\partial k_{z}}{\partial z}\right\rangle-\left|\left\langle\frac{\partial k_{z}}{\partial z}, k_{z}\right\rangle\right|^{2}\right] \\
& \geq 0 .
\end{aligned}
$$

Similarly, we can write $H(z, w)=\pi^{2}\left\langle\delta_{z}, \delta_{w}\right\rangle$, where $\delta_{z}$ denotes the Dirac measure at $z$, from which the polysubharmonicity of $H$ follows.

The remaining statements in the theorem follow from the identity

$$
\Delta f(u)=f^{\prime \prime}(u)|\nabla u|^{2}+f^{\prime}(u) \Delta u
$$

valid for smooth functions $f$ (on $\mathbb{R}$ ) and $u$ (in $\mathbb{R}^{n}$ ). Choosing here $u=E$ and $f(u)=\log (1-u)$ we have $f^{\prime}<0, f^{\prime \prime}<0$, showing that (8.3) implies (8.1). Similarly, choosing $u=1 / H, f(u)=\log u$ shows that (8.2) implies $\Delta \log H \geq 0$.

As a corollary of (8.3) we get the Ahlfors-Beurling estimate [1] for the capacity of $K=\bar{\Omega}$. That capacity, $\operatorname{Cap}(K)$, is defined in terms of the equilibrium potential of $K$ or (minus) the Green's function $G$ of $\mathbb{R}^{2} \backslash K=\mathbb{C} \backslash K$ at infinity via

$$
G(z)=-\log |z|+\log \operatorname{Cap}(K)(K)+O\left(|z|^{-1}\right) \quad(|z| \rightarrow \infty) .
$$

For the exponential transform we have directly from the definition

$$
1-E_{\Omega}(z, z)=\frac{|\Omega|}{\pi|z|^{2}}+O\left(\frac{1}{|z|^{3}}\right) \quad(|z| \rightarrow \infty),
$$

hence

$$
\frac{1}{2} \log \left(1-E_{\Omega}(z, z)\right)=-\log |z|+\log \sqrt{\frac{|\Omega|}{\pi}}+O\left(\frac{1}{|z|}\right) .
$$


Since $G(z)$ can be characterized as the largest negative subharmonic function in $\mathbb{C} \backslash K$ with the singularity $-\log |z|$ at infinity, and $\frac{1}{2} \log \left(1-E_{\Omega}(z, z)\right)$ by $(8.3)$ and (8.8) is a competing function in this respect we have

$$
\frac{1}{2} \log \left(1-E_{\Omega}(z, z)\right) \leq G(z)(z \notin \bar{\Omega}),
$$

and so

$$
\sqrt{\frac{|\Omega|}{\pi}} \leq \operatorname{Cap}(K) .
$$

Assuming $|\partial \Omega|=0$, so that $|\Omega|=|K|$, this is the Ahlfors-Beurling estimate [1].

In higher dimension we similarly get $1-E_{\Omega}(x) \leq u(x)$, where $u$ is the equilibrium potential of $K=\bar{\Omega}$, normalized to be one on $\partial \Omega$ and vanishing at infinity. However, in this case we do not immediately get any estimate of the capacity $\operatorname{Cap}(K)$ because the leading terms in the expansions at infinity are of different orders of magnitude:

$$
\begin{gathered}
u(x)=\frac{\operatorname{Cap}(K)}{(n-2)\left|S^{n-1}\right|} \frac{1}{|x|^{n-2}}+O\left(\frac{1}{|x|^{n-1}}\right), \\
1-E_{\Omega}(x)=\frac{2|\Omega|}{\left|S^{n-1}\right|} \frac{1}{|x|^{n}}+O\left(\frac{1}{|x|^{n+1}}\right) .
\end{gathered}
$$

We see that we would rather like to compare $\left(1-E_{\Omega}\right)^{(n-2) / n}$ with $u$, and it is natural to conjecture that $\Delta\left(1-E_{\Omega}\right)^{(n-2) / n} \geq 0$ when $n \geq 3$. We may remark that when this (conjectured) inequality is written out on a form analogous to (8.5) it becomes

$$
\left(1+\frac{2}{n} \frac{1}{\exp \left[\frac{2}{\left|S^{n-1}\right|} \int \frac{\rho(y) d y}{|y|^{n}}\right]-1}\right)\left|\int \frac{y \rho(y) d y}{|y|^{n+2}}\right|^{2} \leq \frac{\left|S^{n-1}\right|}{n} \int \frac{\rho(y) d y}{|y|^{n+2}},
$$

to be valid for any $0 \leq \rho \leq 1$ vanishing in a neighbourhood of the origin. The case $n=2$ in this inequality corresponds to (8.3) and is therefore known to be true.

In the context of comparing exponential transforms with Newtonian potentials we finally point out that the inequality between geometric and arithmetic means applied to $M_{0}$ and $M_{1}$ at the end of Section 3 gives the comparison

$$
\frac{1}{H_{\Omega}(x)} \leq 2(n-2) U^{\Omega}(x) \quad(x \in \Omega)
$$

for convex domains $\Omega$. 
8.1. Acknowledgement. First author was supported by the Swedish Research Council; second author was partially supported by the National Science Foundation Grant DMS 0100367.

\section{REFERENCES}

[1] L. AhlFors \& A. BEURling, Conformal invarinats and function-theoretic null-sets, Acta Math. 83 (1950), 101-129.

[2] D. AHARONOV \& H.S. SHAPIRO, Domains on which analytic functions satisfy quadrature identities, J. Analyse Math. 30 (1976), 39-73.

[3] N.I. AkHiEZer, The Classical Moment Problem and Some Related Questions in Analysis, Oliver and Boyd, Edinburgh/London, 1965.

[4] S. BERGMAN \& M. SCHIFFER, Kernel functions and conformal mapping, Compositio Math. 8 (1951), 205-249.

[5] R.W. CAREY \& J.D. PinCUS, An exponential formula for determining functions, Indiana Univ. Math. J. 23 (1974), 1031-1042.

[6] PH.J. Davis, The Schwarz function and its applications, Carus Math. Mono. vol. 17, Math. Assoc. Amer., 1974.

[7] H. Flanders, Differential Forms with Applications to the Physical Sciences, Academic Press, New York, 1963.

[8] R.J. Gardner, Geometric Tomography, Cambridge Univ. Press, 1995.

[9] B. GustafsSOn, On mother bodies of convex polyhedra, SIAM J. Math. Anal. 29:5 (1998), 1106-1117.

[10] B. GUSTAFSSON \& M. PUTINAR, An exponential transform and regularity of free boundaries in two dimensions, Ann. Sc. Norm. Sup. Pisa 26 (1998), 507-543.

[11] _ Analytic continuation of Cauchy and exponential transforms, In: Analytic extension Formulas and their Applications (S. Saitoh et al., eds.), Kluwer Academic Publishers, Netherlands, 2001 pp. 47-57.

[12] B. Gustafsson, C. He, P. Milanfar \& M. Putinar, Reconstructing planar domains from their moments, Inverse Problems 16 (2000), 1053-1070.

[13] R. HARVEY \& B. LAWSON, Boundaries of complex analytic varieties, Bull. Amer. Math. Soc. 80 (1974), 180-183.

[14] F. JOHN, Plane Waves and Spherical Means Applied to Partial Differential Equations, Interscince Publishers, Inc., New York, 1955.

[15] M.G. Krein \& A.A. Nudelman, Markov Moment Problems and Extremal Problems, Translations of Math. Monographs Volume 50, Amer. Math. Soc., Providence, RI, 1977.

[16] N.S. LANDKof, Foundations of Modern Potential Theory, Springer-Verlag, Berlin-Heidelberg, 1972

[17] M. Martin \& M. PUtinar, Lectures on Hyponormal Operators, Birkhäuser, Basel, 1989.

[18] J.D. PINCUS \& J. ROVNYAK, A representation formula for determining functions, Proc. Amer. Math. Soc. 22 (1969), 498-502.

[19] M. PUTINAR, Extremal solutions of the two-dimensional L-problem of moments, J. Funct. An. 136 (1996), 331-364.

[20] _ Extremal solutions of the two-dimensional L-problem of moments II, J. Approx. Th. 92 (1998), 38-58. 
[21] M. RIESZ, Lintégrale de Riemann-Liouville et le problème de Cauchy, Acta Math. 81 (1951), $1-223$.

[22]__ Intégrales de Riemann-Liouville et potentiels, Acta Szeged Sect. Math. 9 (1937-38), 1-42.

[23] M. SAKAI, Regularity of boundary having a Schwarz function, Acta Math. 166 (1991), 263-297.

[24] H.S. SHAPIRO, The Schwarz function and its generalization to higher dimensions, Uni. of Arkansas Lect. Notes Math. Volume 9, Wiley, New York, 1992.

[25] E. Stein, Singular Integrals and Differentialbility Properties of Functions, Princeton University Press, Princeton, 1970.

[26] F.W. WARnER, Foundations of differentiable Manifolds and Lie groups, Scott, Foremans and Co., Glenview, 1971.

ACKNOWLedgment: The first author was supported by the Swedish Research Council. The second author was partially supported by the National Science Foundation Grant DMS 0100367

BJÖRN GUSTAFSSON:

Mathematics Department

Royal Institute of Technology

S-10044 Stockholm, Sweden.

E-MAIL: gbjorn@math.kth.se

Mihai Putinar:

Department of Mathematics

University of California

Santa Barbara, CA 93106, U. S. A.

E-MAIL: mputinar@math.ucsb.edu

KEY WORDS AND PHRASES: Author: Please supply.

2000 Mathematics Subject Classification: 31C05, 44-99, 44A12, 53C65.

Received: April 10th, 2002. 